Revue des patrimoines

$31 \mid 2017$

Patrimoines de la santé : essais de définition - enjeux de conservation

\title{
La collection de machines orthopédiques de François Humbert (1776-1850), médecin meusien, fondateur du premier établissement orthopédique français
}

The collection of orthopaedic machines of François Humbert, doctor in the Meuse department and founder of the first orthopaedics centre in France

Antoine Desseaux, Étienne Guibert, Michel Pionnier et Marguerite Préau Sido

\section{(2) OpenEdition}

Journals

Édition électronique

URL : http://journals.openedition.org/insitu/14064

DOI : $10.4000 /$ insitu. 14064

ISSN : 1630-7305

Éditeur

Ministère de la Culture

Référence électronique

Antoine Desseaux, Étienne Guibert, Michel Pionnier et Marguerite Préau Sido, «La collection de machines orthopédiques de François Humbert (1776-1850), médecin meusien, fondateur du premier établissement orthopédique français », In Situ [En ligne], 31 | 2017, mis en ligne le 22 février 2017, consulté le 09 octobre 2020. URL : http://journals.openedition.org/insitu/14064 ; DOI : https://doi.org/ 10.4000/insitu. 14064

Ce document a été généré automatiquement le 9 octobre 2020.

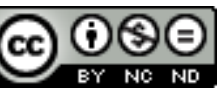

In Situ Revues des patrimoines est mis à disposition selon les termes de la licence Creative Commons Attribution - Pas d'Utilisation Commerciale - Pas de Modification 4.0 International. 


\section{La collection de machines} orthopédiques de François Humbert (1776-1850), médecin meusien, fondateur du premier établissement orthopédique français

The collection of orthopaedic machines of François Humbert, doctor in the Meuse department and founder of the first orthopaedics centre in France

Antoine Desseaux, Étienne Guibert, Michel Pionnier et Marguerite Préau Sido

\section{Introduction}

1 François Humbert, simple officier de santé pendant la première moitié du XIXe siècle, fonde à Morley (Meuse) en 1817 le premier établissement orthopédique de France. Passionné, il conduit ses recherches médicales et devient le premier à ouvrir la voie de la prise en charge des luxations congénitales de la hanche, réputées incurables. La teneur de ses travaux se répand rapidement, provoquant admiration et débats houleux. Isolé, sans héritier, cet orthopédiste tombe dans l'oubli après sa mort. Il laisse cependant plusieurs témoignages de cette activité médicale qui connaissent un parcours mouvementé.

2 Aujourd'hui, l'ancien établissement, converti en maisons d'habitation, se dresse toujours dans le village. Dispersée, une partie de sa collection est entrée au Musée barrois de Bar-le-Duc (Meuse) où se trouve une quarantaine de maquettes orthopédiques de son invention. Fruit d'un partenariat entre le Musée barrois et la récente association François Humbert de Morley, ses modèles réduits font l'objet d'une réflexion muséologique, tant sur leur conservation que sur leur future mise en valeur. 


\section{François Humbert, fondateur du premier établissement orthopédique français}

\section{Une formation médicale difficile}

3 François Humbert naît à la veille de la Révolution française, en 1776, à Châlons-enChampagne, dans une famille de magistrats où rien ne le prédisposait à embrasser une carrière médicale. Son grand-père paternel est avocat à la cour et maire royal d'Ancerville (Meuse) et son grand-père maternel est procureur à Châlons-enChampagne (Marne). Au gré des mésententes familiales et des épisodes révolutionnaires, il suit des études primaires chaotiques. Il qualifie lui-même sa première instruction de médiocre. Cependant, dès l'enfance, il montre une passion pour la mécanique, en reproduisant, par exemple, les installations de la sonnerie du clocher de la cathédrale de la ville ${ }^{1}$.

En 1794, sa famille l'oblige à entrer à l'hôpital militaire ambulant de Châlons-enChampagne. Bien qu'il s'y engage à contrecœur², il obtient le grade de chirurgien de troisième classe et se passionne pour l'étude de l'anatomie.

En 1796, un arrêté licencie tous les chirurgiens non commissionnés des Armées. Libéré, il s'installe à Paris où il devient étudiant à l'École de médecine. Sans argent, il suit les cours dispensés gratuitement, comme ceux de physique expérimentale de Louis Lefèvre-Gineau, ou ceux d'anatomie d'Antoine Portal au Collège de France et les leçons d'anatomie comparée de Georges Cuvier, dispensées au Muséum national d'histoire naturelle ${ }^{3}$. Pour vivre, il occupe les fonctions de prosecteur dans un amphithéâtre d'anatomie ouvert dans les locaux de la Sorbonne.

Lors de la deuxième campagne d'Italie, il s'engage à nouveau dans l'armée. Bien que devenu chirurgien de deuxième classe, il prend conscience, après quelques mois, que sa carrière militaire ne lui permettra ni d'approfondir ses connaissances médicales, ni d'assouvir sa curiosité scientifique. Par conséquent, il démissionne en septembre 1800.

7 De retour dans l'est de la France, il est reçu comme officier de santé dans le département de la Meuse, où il s'installe dans un petit village, Morley. Épousant sa cousine, Jeanne de Fleury, fille d'un notable, il jouit rapidement d'une notoriété locale. Parallèlement à son activité de médecin de campagne, il est nommé en 1805 au poste de chirurgien en chef de l'hospice civil et militaire de Joinville (Haute-Marne). Il y reste jusqu'en 1810, date à laquelle il démissionne pour reprendre ses fonctions médicales à Morley. C'est dans ce village isolé qu'il ouvre son établissement orthopédique.

\section{L'établissement de Morley : premier établissement orthopédique de France}

En 1816, à l'heure où sa clientèle d'officier de santé diminue fortement du fait d'une concurrence nouvelle due aux licenciements d'une partie des médecins de l'armée, François Humbert réfléchit à de nouvelles activités plus en lien avec ses aspirations.

Dans un premier temps, il pense ouvrir un établissement de bains de vapeur, la rivière du village, la Saulx, pouvant l'approvisionner facilement en eau. Mais au hasard de sa patientèle, il reçoit une jeune personne souffrant d'un renversement du bassin. Après 
de nombreuses hésitations, il commence un traitement, usant à la fois de bains de vapeur et de moyens mécaniques de son invention. Après quelques semaines de soins, la patiente commence à marcher sans claudication. Encouragé par le docteur Champion, médecin reconnu de Bar-le-Duc, il continue à réfléchir à des procédés permettant le traitement des incurvations spinales ${ }^{4}$.

Fort de ce premier succès et des soutiens reçus, il ouvre officiellement en 1817 l'établissement orthopédique de Morley (fig. 1). Il fonde ainsi le premier établissement de ce type en France. Fier de ce prestige, l'épitaphe gravée sur sa pierre tombale, encore visible au cimetière de Morley, le mentionne.

Figure 1

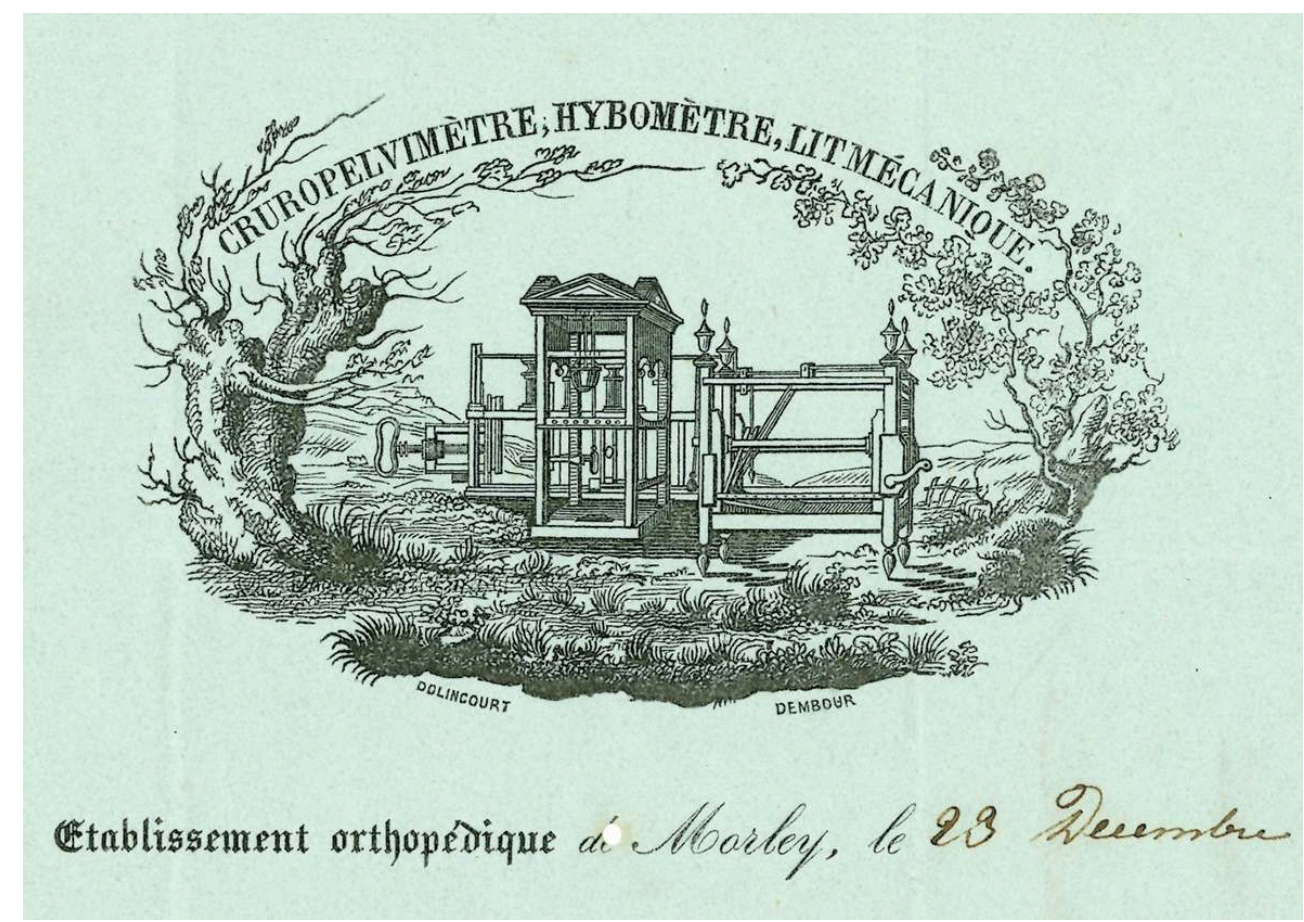

Papier à en-tête de l'établissement orthopédique de Morley, 1850 (détail). Musée barrois, Documentation, dossier François Humbert.

(c) Musée barrois, Bar-le-Duc, 2015.

11 L'ouvrage du docteur T. Rapou ${ }^{5}$, de Lyon, qui annonce avoir corrigé une déviation de la colonne vertébrale par l'usage de douches et de bains de vapeur, vient le conforter dans ses recherches. Ingénieux mécanicien, François Humbert reste persuadé que l'utilisation d'appareils mécaniques est une approche pertinente. Les traitements dispensés à Morley allient les deux procédés :

«Pour obtenir des succès en orthopédie, des connaissances approfondies, et une grande pratique en médecine et en chirurgie, ne sont pas suffisantes; il faut encore des dispositions innées pour la mécanique. Un ouvrier, si habile et si intelligent qu'il puisse être, se pénétrera difficilement de la pensée des médecins, parce qu'il ne connaît pas lui-même la médecine, et que, par conséquent, il ne pourra pas bien saisir les indications à remplir. Pour arriver à ce résultat, il faut qu'il y ait chez le même individu réunion des deux genres de connaissances. On a donc tort de considérer l'orthopédie comme une division de la chirurgie, pour laquelle on doit, ainsi que pour toutes les autres, abandonner entièrement à des artistes tout ce qui concerne les machines et appareils. Tout au plus s'il en serait ainsi, en admettant que les difformités puissent être corrigées par un nombre de machines limité et 
donc la construction serait toujours la même... Elles sont aussi variées que les cas auxquels elles s'appliquent, et tous les jours on peut se trouver dans la nécessité d'ajouter à leurs modifications. Je ne connais pas d'orthopédie possible, si elle n'est dirigée dans cet esprit $»^{6}$.

L'établissement de Morley traite pour l'essentiel des patientes atteintes de scoliose ou de luxation de hanche (congénitale ou non). Ces pathologies, plus fréquentes chez les filles, et les représentations médicales de l'époque sur la maternité 7 , expliquent, comme la plupart des établissements de l'époque, que la maison de Morley soit réservée aux jeunes filles. Bien que retiré, cet établissement connaît un véritable essor. En effet, plusieurs articles parus dans la presse nationale lui assurent une véritable publicité. (Le Constitutionnel $^{8}$, Le Journal des débats $\left.{ }^{9}\right)$. Malgré l'isolement géographique du village, les patientes affluent. En dix ans, François Humbert annonce avoir accueilli plus de deux cents patientes ${ }^{10}$. Les visiteurs parlent en général d'une vingtaine de jeunes filles présentes en même temps à Morley. Elles sont âgées de treize à dix-neuf ans ${ }^{11}$ et certaines viennent de pays éloignés, comme le mentionne une lettre du conseil municipal de Morley datée du 21 juin 1835. « Nous vîmes arriver des familles de la plus haute distinction, non seulement de tous les coins de la France, mais encore des pays étrangers, tels que la Suisse, la Bavière, la Transylvanie, les Pays-Bas, l'Écosse, l'Irlande, l'Angleterre et le Portugal $»^{12}$.

En 1834, alors que l'établissement de Morley est à son apogée, François Humbert en donne une description globale (fig. 2, 3, 4, 5).

Figure 2

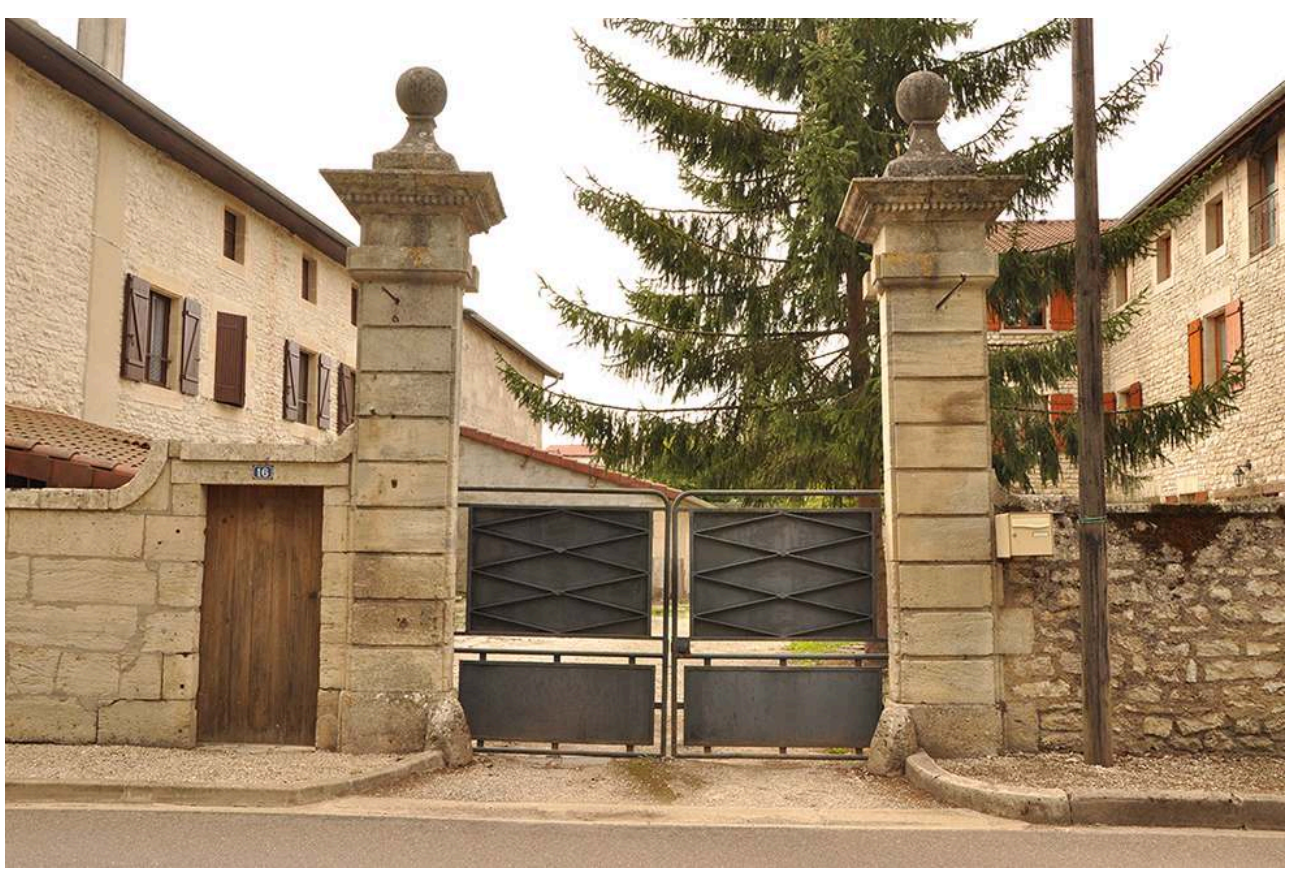

Portail central de l'établissement orthopédique de Morley, construit vers 1820. État actuel, rue du Docteur Humbert, 55290 Morley.

(C) Association F. Humbert de Morley, 2015. 
Figure 3

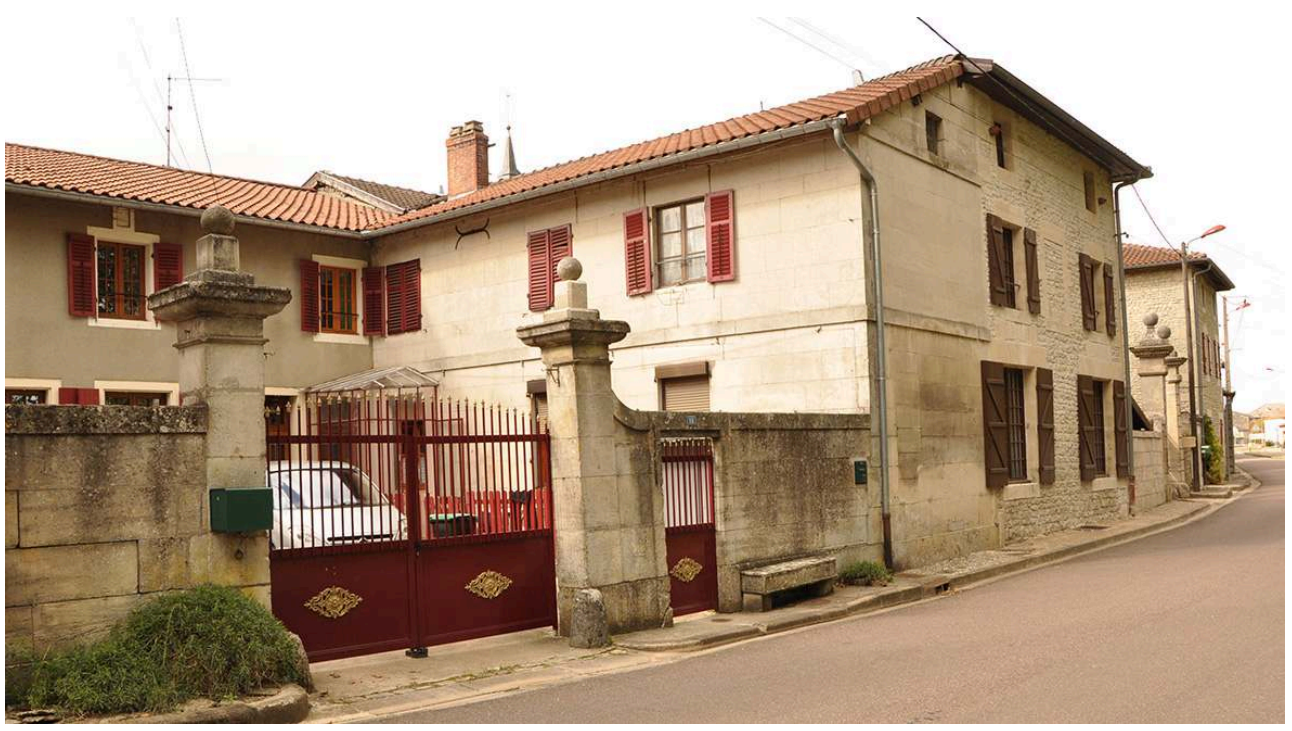

Façade de l'établissement orthopédique de Morley. État actuel, rue du Docteur Humbert, 55290 Morley.

(c) Association F. Humbert de Morley, 2015.

\section{Figure 4}

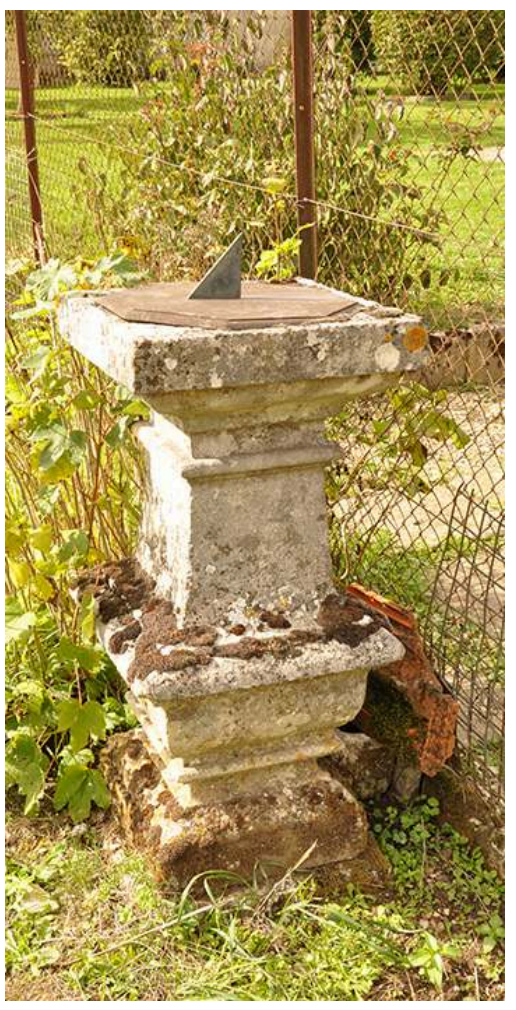

Cadran solaire, jardin au levant, établissement orthopédique de Morley vers 1830. État actuel, jardin privé, rue du Docteur Humbert, 55290 Morley.

(c) Association F. Humbert de Morley, 2015. 
Figure 5

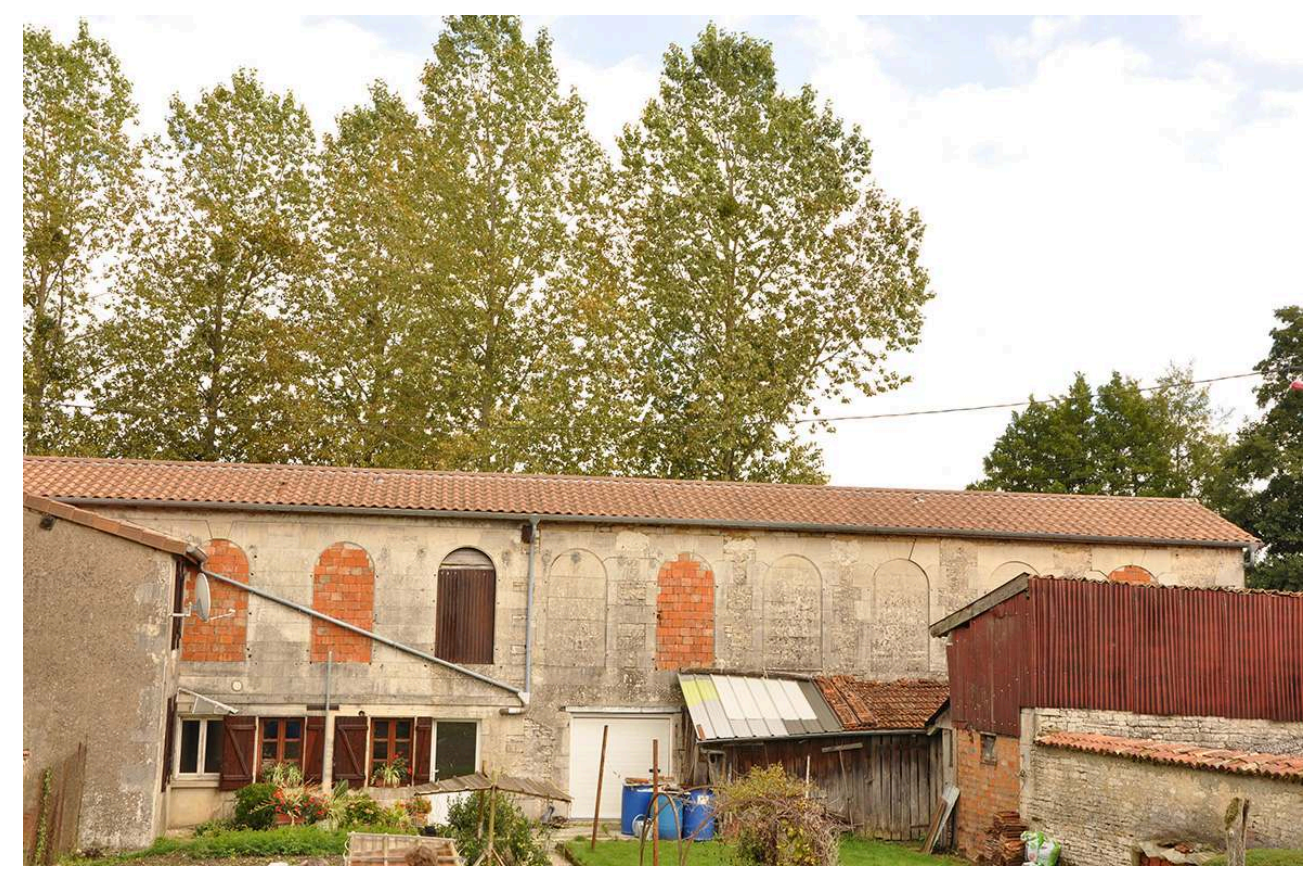

Façade du théâtre de l'établissement orthopédique de Morley, côté jardin du levant, 1826. État actuel, rue du Docteur Humbert, 55290 Morley.

(c) Association F. Humbert de Morley, 2015.

«À ces avantages immenses, dus aux connaissances spéciales de son fondateur, l'établissement de Morley joint encore ceux de la situation. Construite sur les bords de la rivière de Saulx, dont la vallée est agréable, cette maison fait partie du village de Morley; une forêt aussi belle qu'elle est étendue, couronne le coteau situé au couchant. L'air de cette contrée est pur et assez vif. Les bâtiments de l'établissement ont été étendus d'années en années en raison des besoins, et maintenant ils présentent un développement de trois cent cinquante pieds au levant sur cent cinquante pieds de profondeur. Un jardin au midi et un autre au couchant sur les rives de la Saulx servent de vaste promenade. Des plantations, une serre, un cabinet d'histoire naturelle, une bibliothèque, une salle ou galerie pour la danse, une salle de billard; des bains, des ateliers pour la confection des machines, appareils, corsets, cuirs, etc., ainsi que pour la menuiserie et la serrurerie, et un musée orthopédique forment les dépendances de ce bel établissement $»^{13}$.

Le décès (en 1844) de son fils unique, Nicolas Jules Humbert, docteur en médecine ${ }^{14}$, alors que François Humbert est âgé de 68 ans, est le prétexte à la fermeture définitive, en 1846, de l'établissement de Morley. Il est démantelé au fur et à mesure des ventes à divers particuliers et transformé en maisons d'habitation. Les travaux et l'histoire de ce personnage tombent rapidement dans l'oubli.

\section{Les collections du médecin}

La description donnée de l'établissement orthopédique en 1834 atteste de la curiosité de son fondateur. En plus du cabinet d'histoire naturelle et du musée orthopédique, François Humbert possédait une collection d'antiquités et d'objets d'art.

16 La composition de son cabinet d'histoire naturelle est connue aujourd'hui par des inventaires dressés ${ }^{15}$ à sa mort. Reflet de son intérêt pour l'anatomie, il renfermait plusieurs spécimens d'ostéologie : squelettes humains entiers (adulte, enfant, fœetus) ou 
démontés, comme une colonne vertébrale d'un enfant de 10 à 12 ans. On compte par ailleurs une douzaine de squelettes de quadrupèdes, ainsi qu'une trentaine de squelettes d'oiseaux. Plusieurs fanons de baleine, scies d'espadons et têtes de poissons sont mentionnés dans la liste du don effectué en 1850 au Musée barrois. Onze carapaces de tortues, ainsi qu'une quarantaine de défenses diverses (bois d'antilopes, de rennes, défenses d'éléphant et d'hippopotame, corne de rhinocéros...) et une trentaine de coraux complètent la collection. Ce cabinet d'histoire naturelle ne serait pas complet sans la présence de minéraux : près de trois cents échantillons de pierres, minéraux et plus de cinquante fossiles et autres curiosités naturelles y figurent.

17 François Humbert collectionne par ailleurs de nombreux objets touchant aux principaux domaines intéressant "l'honnête homme " du XIX siècle. Aussi l'histoire naturelle voisine-t-elle naturellement avec les témoignages archéologiques et historiques, les œuvres et objets d'art de toute époque et de tout continent. De cette collection, seuls sont connus les objets donnés aux Musée lorrain de Nancy et au musée de Bar-le-Duc par les héritiers du médecin.

Dans un contexte actif de fouilles locales, auxquelles s'ajoutent de nombreuses découvertes fortuites, l'archéologie gallo-romaine est une composante importante de la collection. En effet, plusieurs découvertes ont été faites sur le territoire même de Morley. François Humbert conservait ainsi de nombreuses pièces, aujourd'hui au Musée barrois : des éléments lapidaires remarquables («Stèle de l'oculiste», inv. 850.20.1 (fig. 6) ; "Lion en ronde-bosse", inv. 850.20.3) et un intéressant mobilier (urne cinéraire, clef et clochette en bronze, vases et fragments de poterie, lampe, médaillon...). 
Figure 6

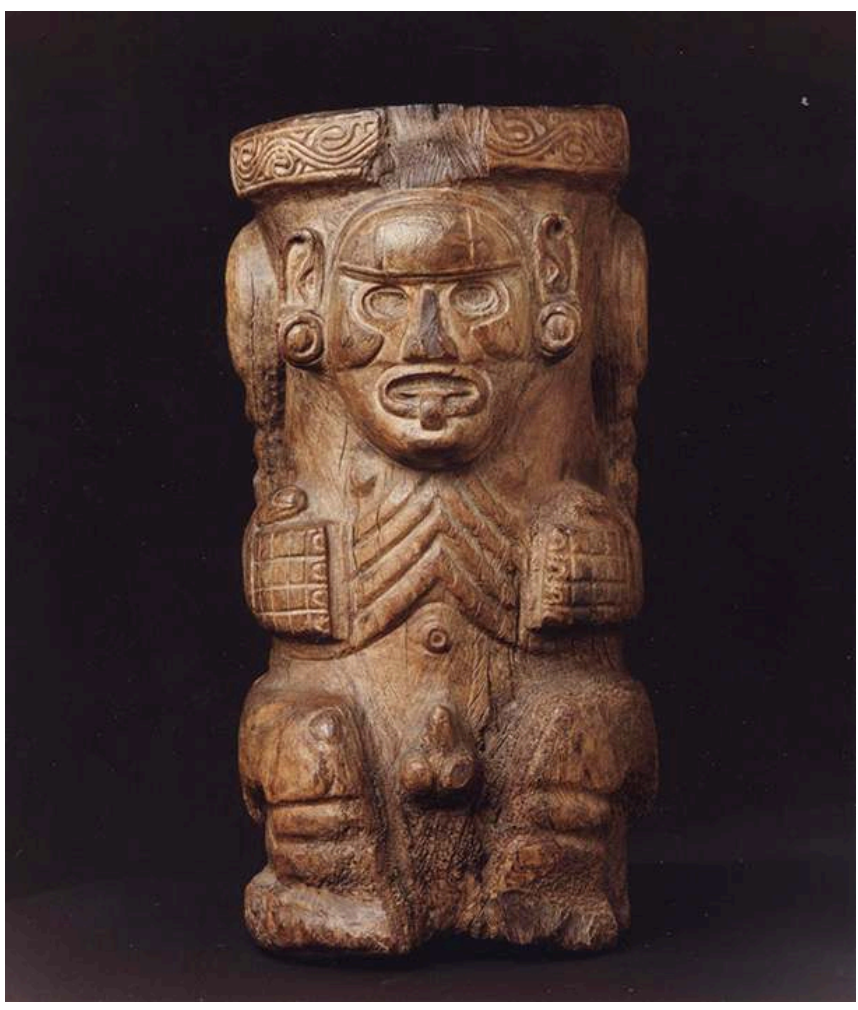

Urne funéraire Taïno. Bois sculpté, Xle ou XIIe siècle. Provenance : Grandes Antilles. Collection du Musée barrois, don de Mme Veuve Humbert, 1850, n inv. 850.20.38.

(c) Musée barrois, Bar-le-Duc, 2009.

La Renaissance et l'époque moderne sont représentées par plusieurs armes, cuirasses, casques, un coffret en bois aux armes de Marie-Antoinette de Lorraine, ainsi qu'un Portrait d'Henri de Lorraine (inv. 850.20.3), l'ensemble provenant de la maison des ducs de Guise, princes de Joinville: François Humbert, actif quelques années à l'hôpital de Joinville, semble en effet avoir gardé plusieurs œuvres et archives de l'établissement qu'il quitta en 1810. Le Musée lorrain conserve par ailleurs un bel émail de Limoges ( $L a$ Mise au tombeau, $\mathrm{XvI}^{\mathrm{e}}$ siècle, inv. 95.747) provenant de la succession Humbert.

Les objets d'art extra-européens, objets de curiosité, sont également bien représentés dans la collection Humbert. Parmi ceux-là, un gobelet en ivoire chinois fut donné au Musée lorrain «en souvenir de la campagne d'Égypte » (inv. 95.202). Au musée de Barle-Duc parviennent plusieurs pièces d'importance variable: une hache indienne, des magots chinois, ainsi qu'un "fétiche égyptien, [...] plutôt d'origine mexicaine, figure grotesque $[. . .]^{16} "$, aujourd'hui considéré comme l'une des pièces majeures du Musée barrois (urne funéraire Taïno, Caraïbes, $\mathrm{XII}^{\mathrm{e}}$ siècle, inv. 850.20.38) (fig. 7). 
Figure 7

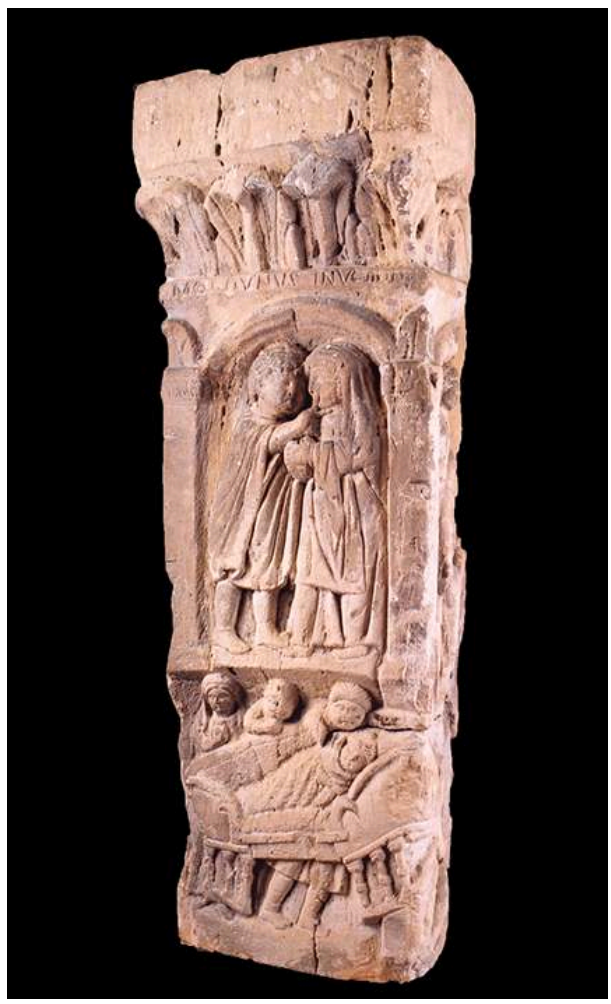

Stèle de l'oculiste. Calcaire sculpté, $7^{\text {er }}$ siècle. Provenance : Montiers-sur-Saulx, fouilles 1829. Collection du Musée barrois, don de Mme Veuve Humbert, 1850, nº inv. 850.20.1.

(c) Musée barrois, Bar-le-Duc, 2015.

Enfin, François Humbert s'intéressait à la numismatique romaine et médiévale, et acheta en 1826 l'important cabinet d'antiques de Jean-Augustin Poëy d'Avant, père du célèbre numismate. Les monnaies furent vendues à sa mort à un particulier ${ }^{17}$.

\section{François Humbert, un médecin de renommée européenne}

François Humbert mène sa carrière médicale au cours d'une période marquée par de profonds changements post-révolutionnaires. La médecine suit l'évolution de la société, passant d'une médecine d'Ancien Régime à une médecine plus moderne. Ainsi, la médecine au chevet des patients laisse progressivement la place à une médecine hospitalière. La spécialisation des médecins conduit à la création de centres ou services spécifiques à certaines pathologies. Le patient est aussi davantage impliqué dans sa prise en charge, avec une relation médecin/malade qui évolue. Les attentes et le ressenti du patient sont davantage pris en considération. Les patients commencent à choisir la structure susceptible de les prendre en charge. Les connaissances médicales ne sont plus l'apanage exclusif des médecins, avec un début de vulgarisation par la publication d'ouvrages et de journaux non médicaux, voire par l'exposé de traitements lors de rencontres publiques ${ }^{18}$. François Humbert écrit en 1838 qu'un traité d'orthopédie, « ouvrage moitié médical, moitié populaire ", pourrait « se répandre dans toutes les classes de la société $»^{19}$. 


\section{Une reconnaissance européenne et institutionnelle}

Le praticien, conscient de l'intérêt de ses travaux, cherche à les faire connaître. Bien que situé dans "un village isolé du monde savant ${ }^{20}$ ", il s'inscrit dans un réseau de sociétés savantes et médicales (Lyon, Marseille, Montpellier, Leipzig, Madrid...) ${ }^{21}$. Il rédige plusieurs ouvrages qu'il diffuse largement, en particulier par l'intermédiaire de la maison d'édition médicale Baillière. Il y décrit les principaux appareils qu'il réalise pour traiter ses patients. Chaque « machine » est adaptée à chaque patient, en partant d'une base commune de lit ou de fauteuil. La diffusion de cette prise en charge novatrice pour l'époque se fait par des livres comme De l'emploi des moyens mécaniques et gymnastiques dans le traitement des difformités du système osseux, dont les deux premiers tomes comportent une succession de schémas permettant de se représenter ou de refaire ces « machines» (fig. 8, 9, 10).

Figure 8

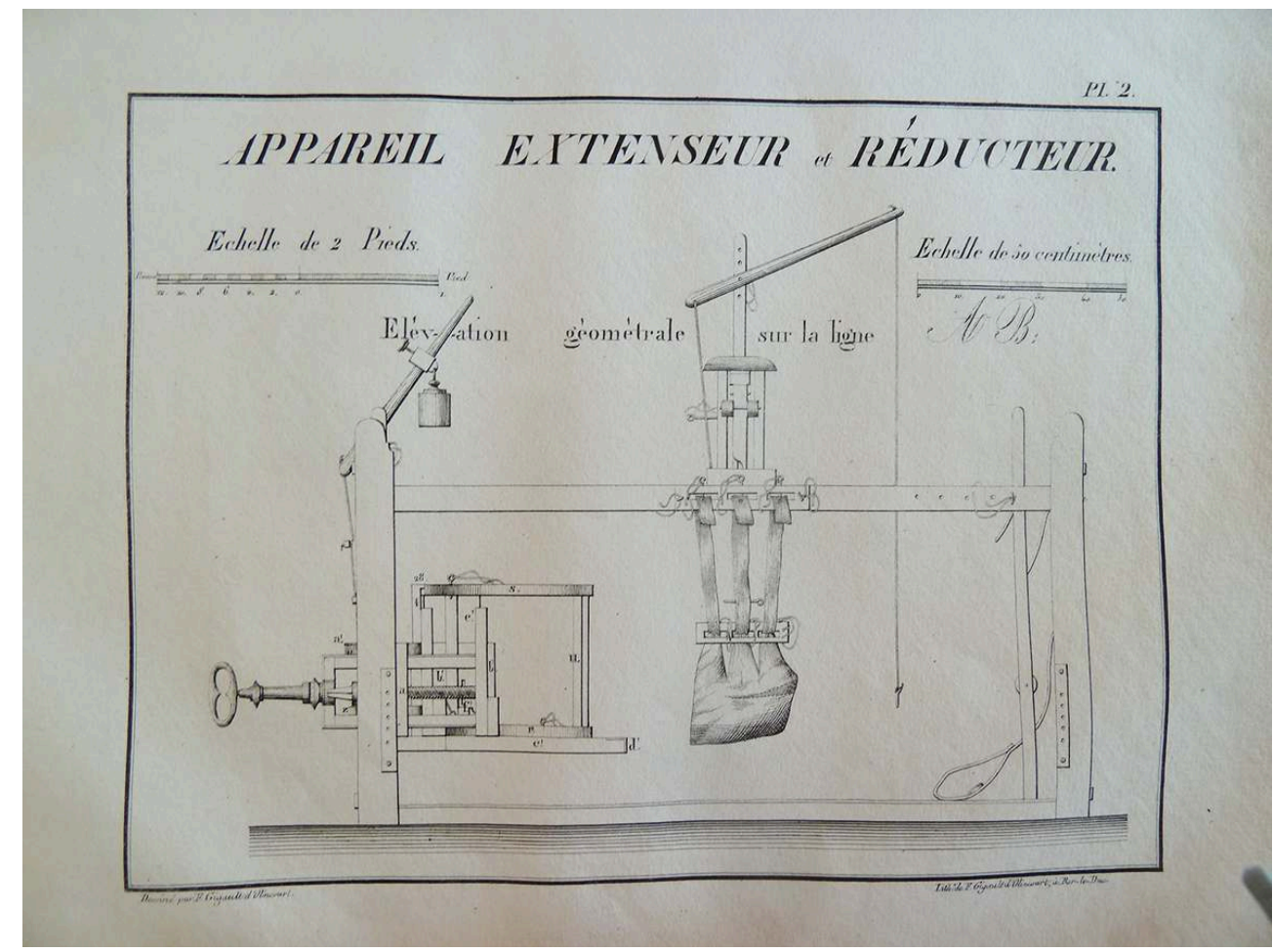

Appareil extenseur et réducteur. Planche extraite de François HUMBERT, Nicolas HUMBERT. « De l'emploi des moyens mécaniques et gymnastiques dans le traitement des difformités du système osseux », 1831-1835.

(C) Association F. Humbert de Morley, 2015. 
Figure 9

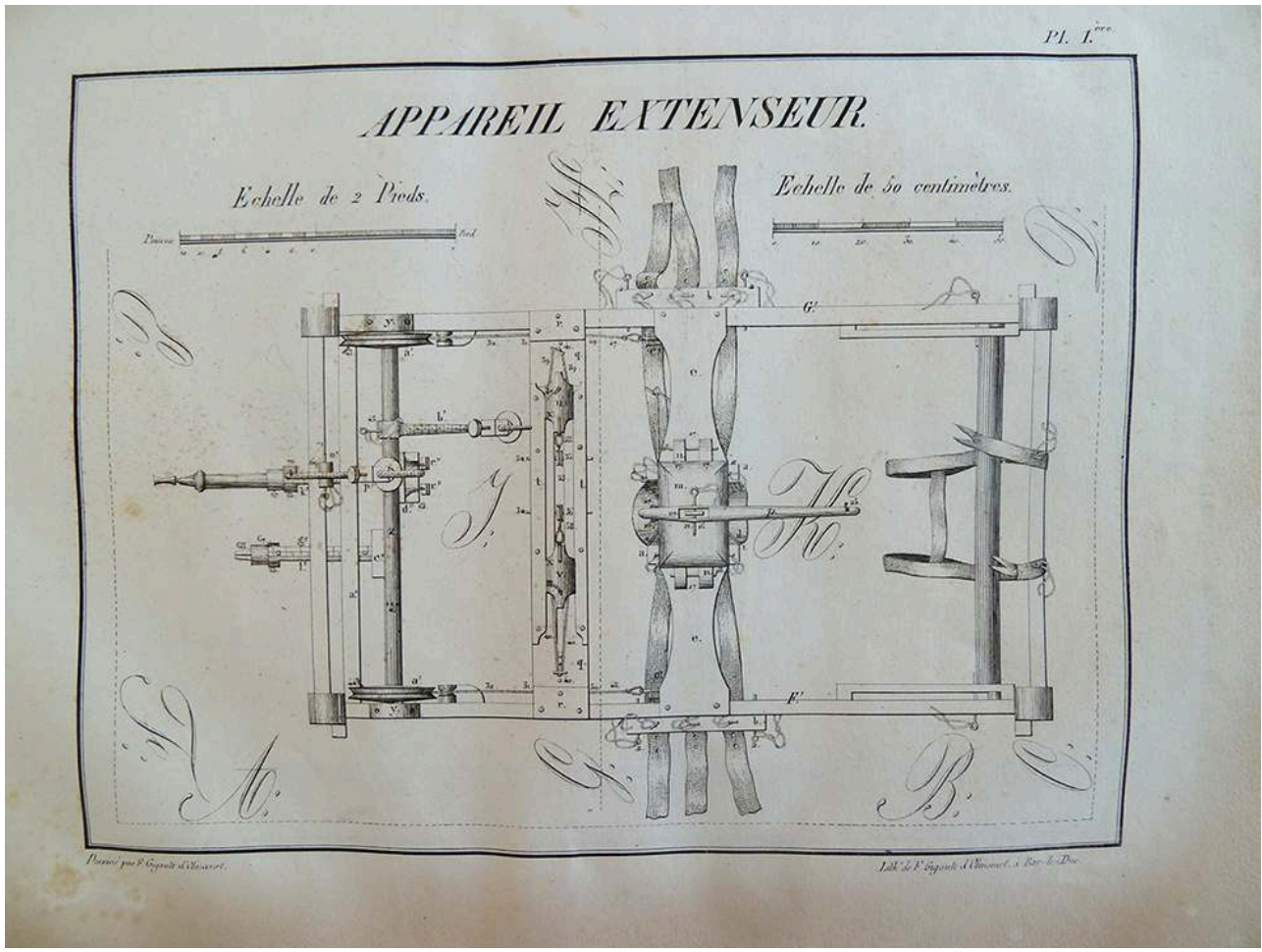

Appareil extenseur. Planche extraite de François HUMBERT, Nicolas HUMBERT. « De l'emploi des moyens mécaniques et gymnastiques dans le traitement des difformités du système osseux », 1831-1835.

(c) Association F. Humbert de Morley, 2015. 
Figure 10

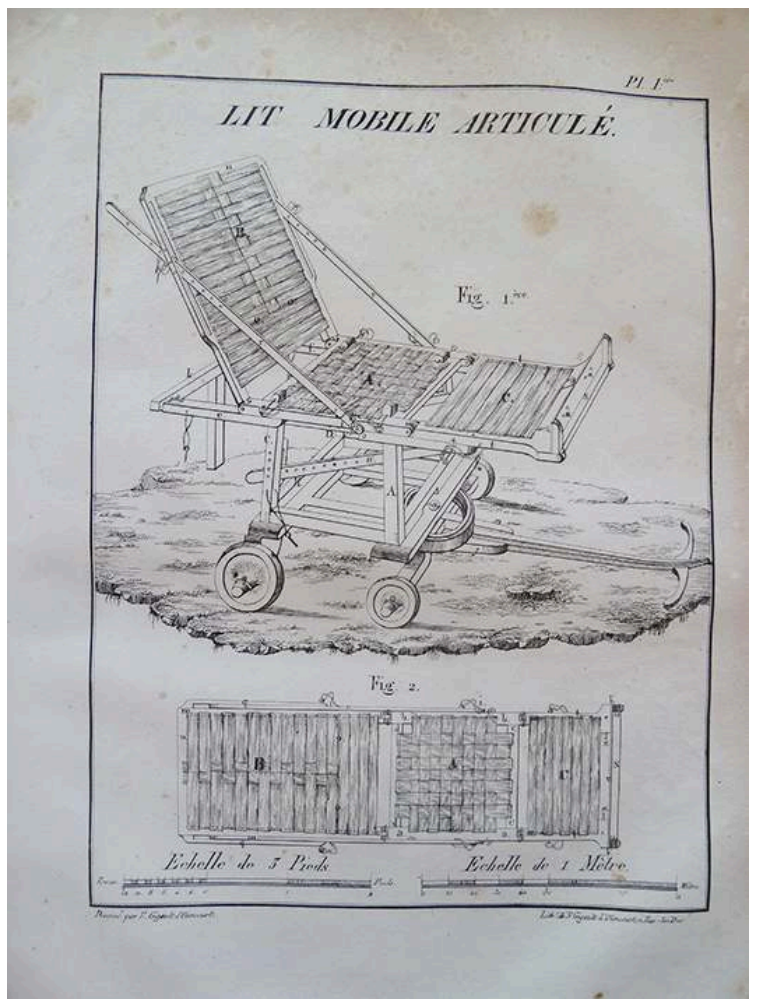

Lit mobile articulé. Planche extraite de François HUMBERT, Nicolas HUMBERT. « De l'emploi des moyens mécaniques et gymnastiques dans le traitement des difformités du système osseux ", 1831-1835.

(c) Association F. Humbert de Morley, 2015

24 Ces ouvrages font l'objet de commentaires plus ou moins élogieux de la part de ses contemporains comme le docteur Charles Gabriel Pravaz, médecin orthopédiste fondateur d'un établissement orthopédique à Lyon, ou les rapporteurs de sociétés médicales et de rédacteurs de journaux médicaux de l'époque ${ }^{22}$. Ses idées franchissent l'Atlantique; The New York Journal of Medicine and Surgery lui consacre un article en juillet 1839. Devant l'impossibilité de conserver et d'exposer de multiples lits ou fauteuils à taille réelle, François Humbert réalise des maquettes de ses "machines». Celles-ci sont conservées dans le musée orthopédique de l'établissement de Morley. Elles permettent de comprendre les positions imposées aux patients ainsi que de se représenter la complexité du montage personnalisé de ces « machines ». 
Figure 11

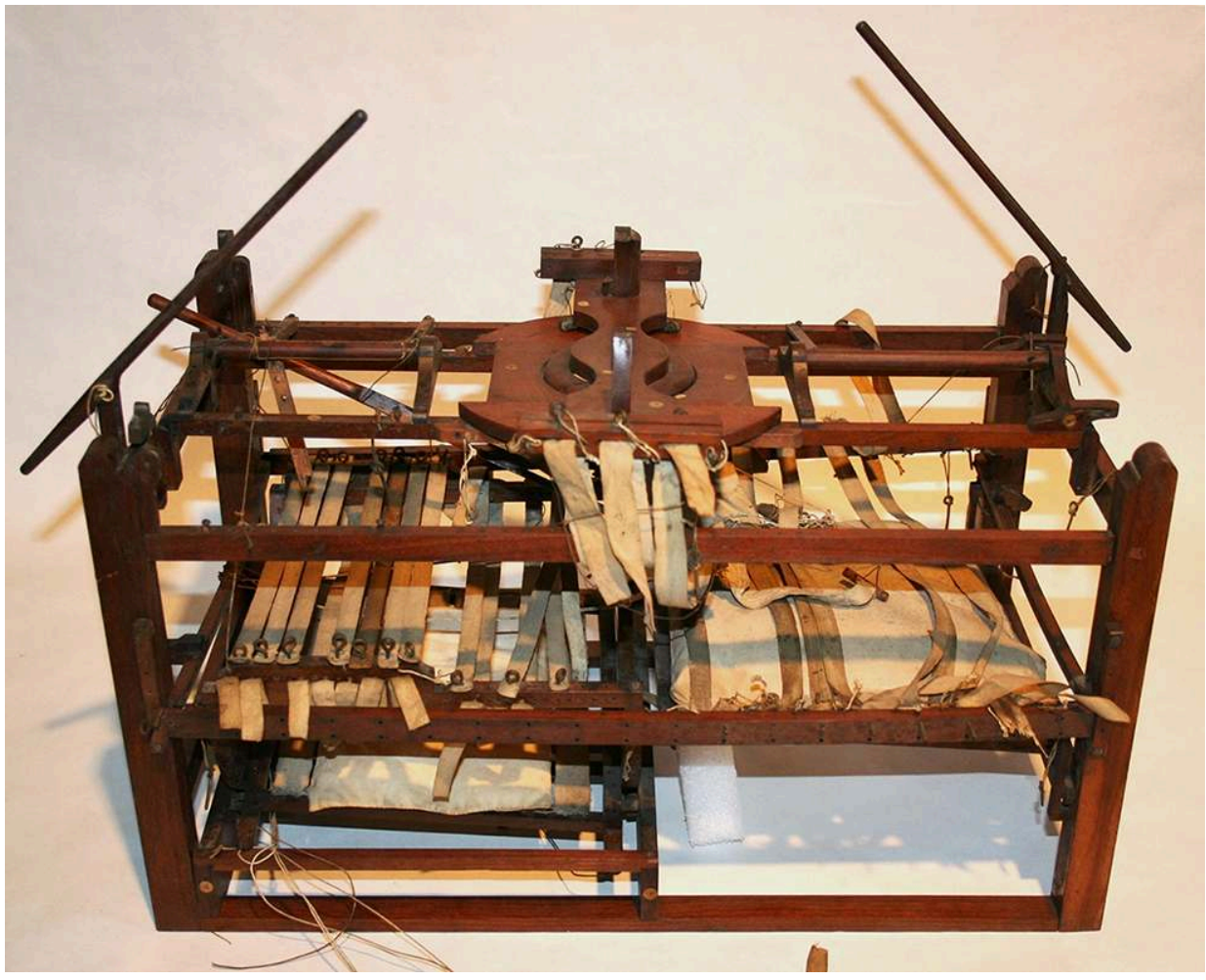

François HUMBERT, Maquette de lit orthopelvien. Bois, textile, corde, métal, vers 1834. Musée barrois, don Humbert, 1850. $n^{\circ}$ inv. prov. 13.10.05.23.

(c) Musée barrois, Bar-le-Duc, 2013 


\section{Figure 12}

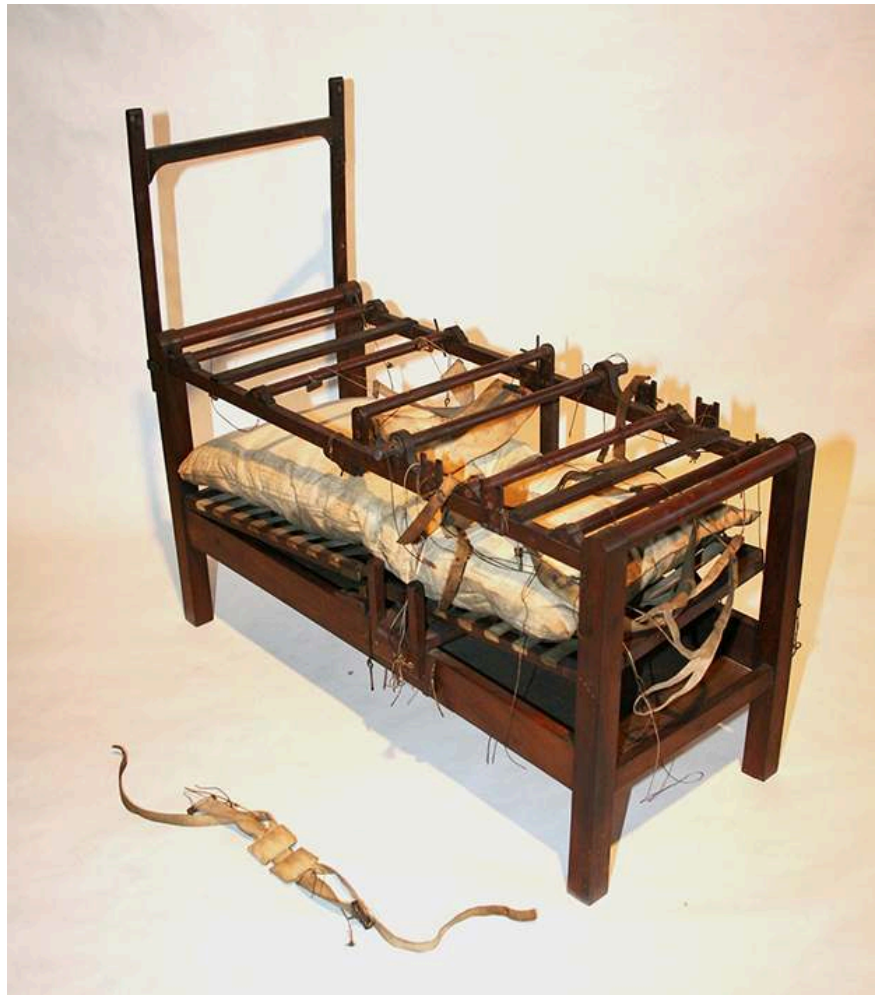

François HUMBERT, Maquette de lit orthorachidique. Bois, textile, corde, métal, vers 1834. Musée barrois, don Humbert, 1850. n inv. prov. 13.10.05.25.

(c) Musée barrois, Bar-le-Duc, 2013. 
Figure 13

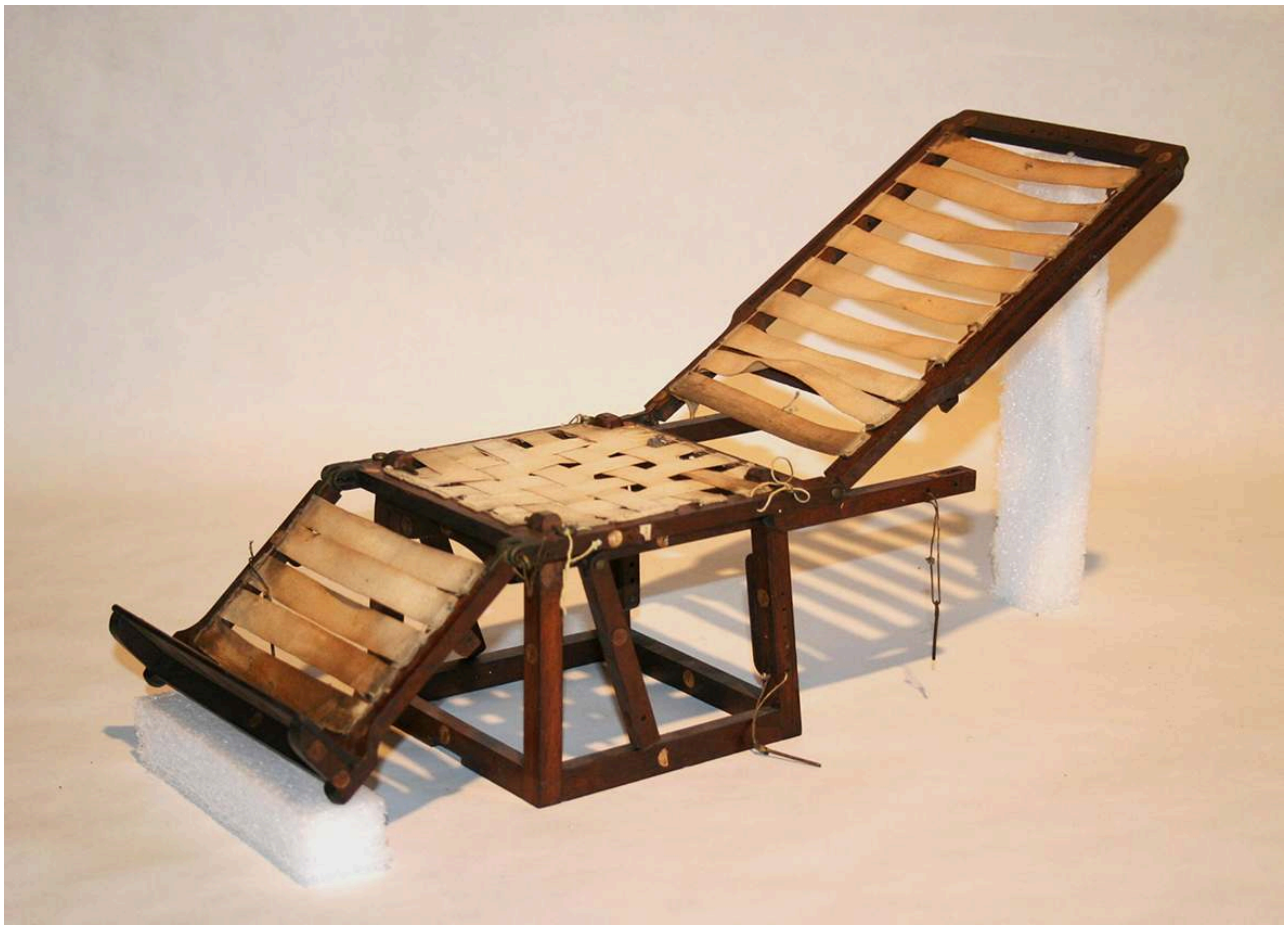

François HUMBERT, Maquette de lit mobile articulé. Bois, textile, corde, métal, vers 1834. Musée barrois, don Humbert, 1850. $n^{\circ}$ inv. prov. 13.10.05.18.

(c) Musée barrois, Bar-le-Duc, 2013.

Le conservateur du Musée barrois en 1850 parle d'une « collection précieuse et unique de modèles de machines, [...] placée dans de superbes verrières ${ }^{23}$ ». Sur les maquettes actuellement conservées au musée, on trouve notamment deux machines de réduction des luxations de hanche, onze lits orthorachidiques (fig. 11, 12, 13), dix fauteuils orthorachidiques (fig. 14, fig. 15), une paire de béquilles, un chariot de promenade, un banc de piano, etc. 
Figure 14

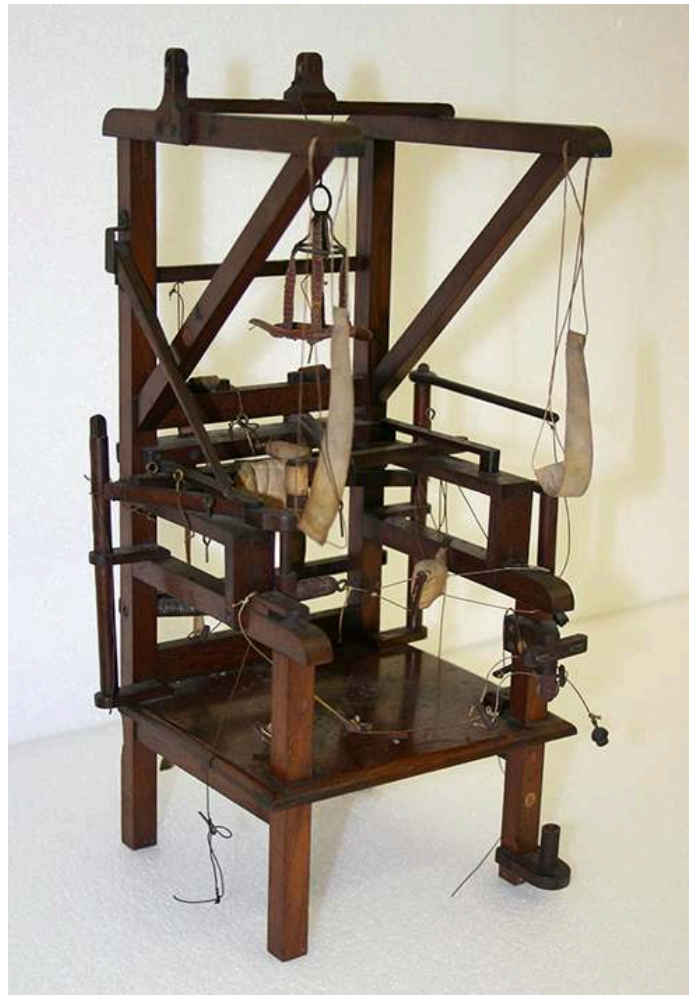

François HUMBERT, Maquette de fauteuil orthorachidique. Bois, textile, corde, métal, vers 1834 Musée barrois, don Humbert, 1850. $n^{\circ}$ inv. prov. 13.10.05.32.

(c) Musée barrois, Bar-le-Duc, 2013 


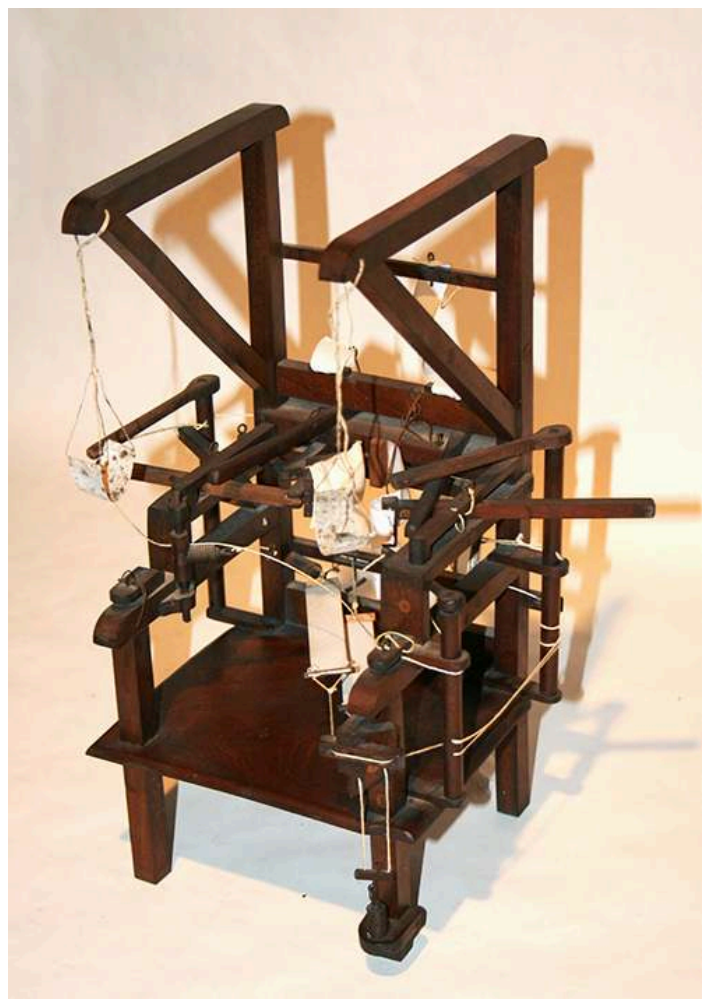

François HUMBERT, Maquette de fauteuil orthorachidique. Bois, textile, corde, métal, vers 1834 Musée barrois, don Humbert, 1850. $n^{\circ}$ inv. prov. 13.10.05.35.

(c) Musée barrois, Bar-le-Duc, 2013 plaît à dire sa « maison ouverte à tous les médecins qui sont venus tout exprès la visiter au moment d'élever des établissements du même genre que le [sien] ${ }^{24}$. Elles permettent aux visiteurs intéressés par cette nouvelle façon de traiter des pathologies de l'époque considérées comme incurables de découvrir les nombreuses possibilités de personnalisation des «machines ». De manière assez ambivalente, François Humbert souhaite «livrer au public la connaissance de tous [ses] procédés » pour éviter que d'autres médecins s'accaparent la primauté de ses techniques de prise en charge ${ }^{25}$. Les maquettes sont également exposées en 1834 à Paris lors de l'Exposition des produits de l'industrie française. La réalisation de modèles transportables de ses «machines » permet aussi à François Humbert, orthopédiste « des bois du département de la Meuse ${ }^{26}$ » de se faire connaître et d'influencer les médecins parisiens. Le désir d'une reconnaissance officielle, notamment à l'occasion du prix Montyon de l'Académie royale des sciences, est probablement le moteur qui a suscité la réalisation des maquettes. François Humbert remporte le prix en $1836^{27}$. Il y présente ses appareils : «tant de machines exécutées en petit, pour modèles, et soumises à l'examen des commissaires de l'Institut, leur transport si difficile et si minutieux ». Ces modèles réduits, de belle facture, sont constitués principalement d'éléments en acajou, de pièces en laiton, tissu et ficelle. François Humbert fait observer le coût élevé de leur exécution, prix de «sacrifices immenses qu'ont dû nécessiter la confection de tant de machines ${ }^{28}$ ».

Il reçoit l'année suivante, en 1837, la Légion d'honneur ${ }^{29}$ pour l'ensemble de son œuvre. 
François Humbert, comme d'autres spécialistes des «déformations» ou anatomistes, réalise des moulages en plâtre ou en cire, afin de garder une "preuve" des modifications apportées. L'un de ses bassins moulés en cire rejoint à Paris le musée Dupuytren $^{30}$ en 1836, sur les conseils du docteur Mathieu Orfila ${ }^{31}$ de la faculté de médecine de Paris.

\section{François Humbert : un orthopédiste précurseur}

"Naturam errantem divina corrigit arte», cette épigraphe rédigée par le doyen de la faculté des lettres de l'académie de Paris, Nicolas-Éloi Lemaire devait être inscrite en dessous d'un portrait de François Humbert ${ }^{32}$; nous pouvons la traduire par «Il corrige par un art divin les défauts de conformation ». Elle exprimait par le mot "divina " le caractère novateur, précurseur et visionnaire de François Humbert dans le redressement des déviations de la nature.

François Humbert propose un protocole thérapeutique avec, en particulier, les lits et fauteuils orthopédiques, mais aussi des appareils de loisirs comme des lits articulés de promenade, montés sur un chariot (fig. 16), des béquilles de promenade, ou encore une table pour écrire (adaptable au fauteuil) (fig. 17). Cette volonté d'adapter l'environnement et les objets au patient évoque les principes de l'ergothérapie.

Figure 16

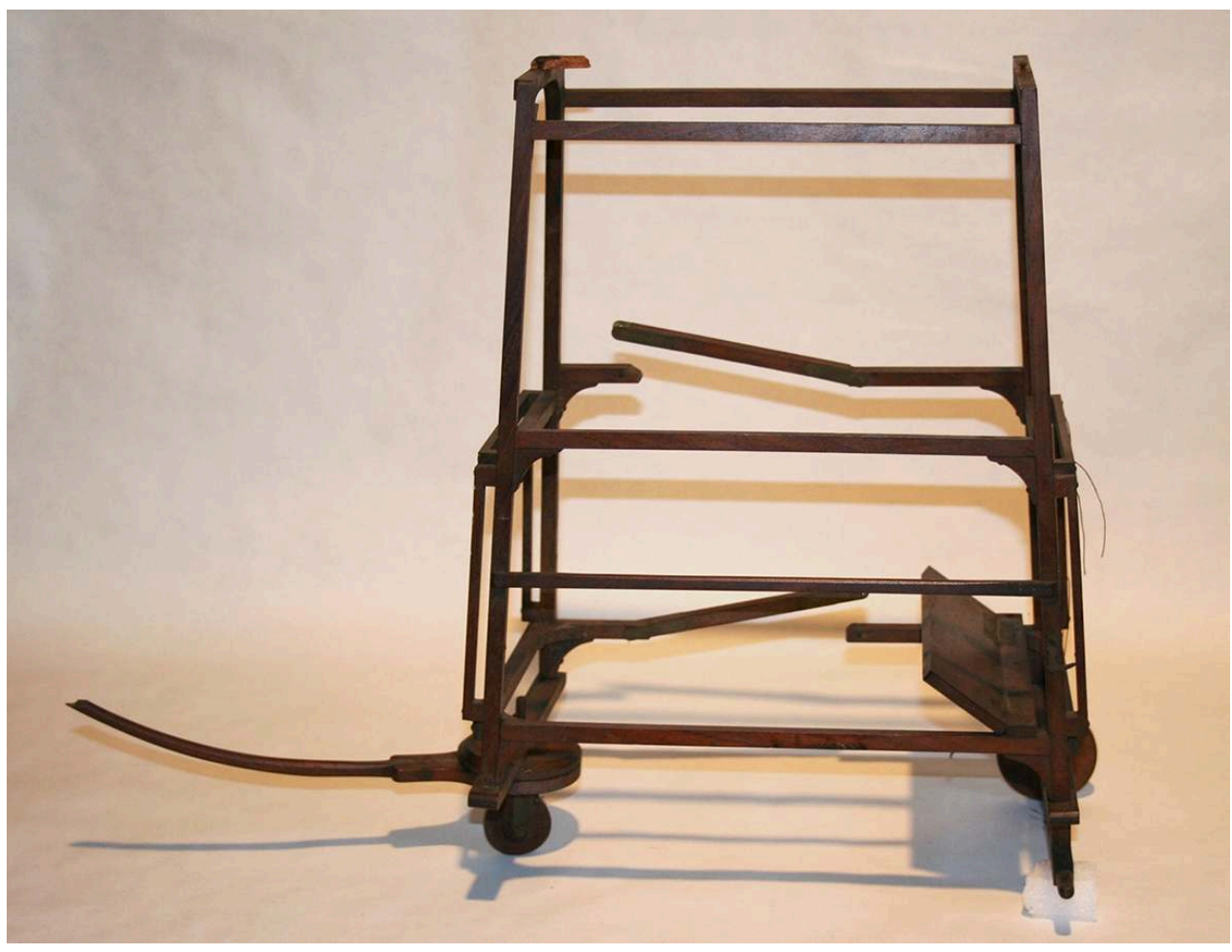

François HUMBERT, Maquette de chariot de promenade. Bois, vers 1834. Musée barrois, don Humbert, 1850. $n^{\circ}$ inv. prov. 13.10.05.17.

(c) Musée barrois, Bar-le-Duc, 2013 


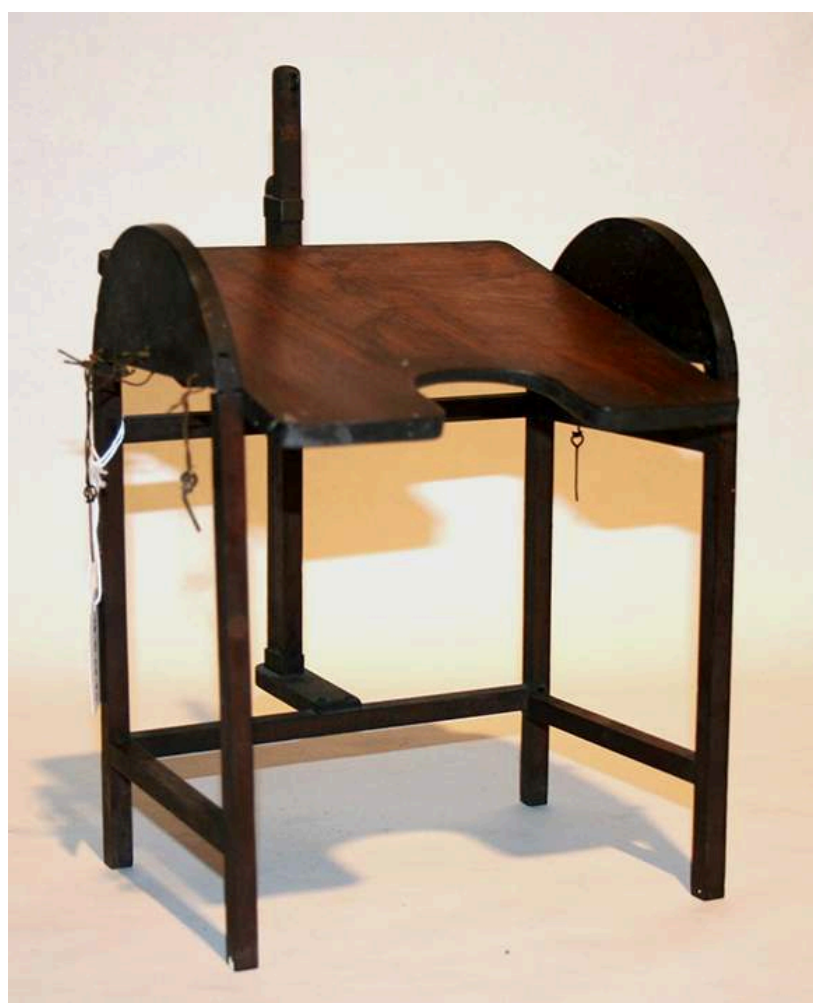

François HUMBERT, Maquette de table de travail. Bois, textile, corde, métal, vers 1834. Musée barrois, don Humbert, 1850. $n^{\circ}$ inv. prov. 13.10.05.34.

(c) Musée barrois, Bar-le-Duc, 2013

L'implication du patient dans sa prise en charge est également forte. Les «forces mécaniques » appliquées sur le patient par des cordes et des poulies sont gérées par le patient lui-même. Il a la possibilité de les moduler et de s'en dégager seul ${ }^{33}$ s'il le souhaite. Ces traitements peuvent durer six à dix-huit mois.

Les ouvrages scientifiques de François Humbert sont à la fois le moyen d'exprimer ses théories et de communiquer ses résultats, voire d'exposer les difficultés rencontrées. Les récits de prise en charge sont très détaillés. Les appareils utilisés le sont également avec des schémas très précis et réalisés à une échelle définie. Il s'oppose aux « expérimentations dangereuses» parfois effectuées selon lui dans d'autres établissements ${ }^{34}$.

François Humbert marque son époque par ses travaux sur la luxation congénitale de hanche (« LCH »). Bien qu'il soigne davantage de patients atteints de scoliose, c'est sa prise en charge des LCH qui lui confère sa renommée. Cette pathologie est un défaut d'emboîtement entre la tête du fémur et le bassin au niveau de la cavité cotyloïdienne. Cela peut conduire à un défaut de croissance du cotyle, voire à une luxation de l'articulation. François Humbert s'intéresse aux formes avancées, c'est-à-dire luxées. Ses traitements reposent sur plusieurs principes, comme les tractions : « Rien ne résiste à ces forces sagement calculées et si, ce qui arrive presque toujours, la nature conserve aux os les rudiments de leurs formes respectives, de manière à ce qu'une fois replacés, ils puissent être maintenus en contact, l'art [vient] seul à bout du reste et les luxations les plus anciennes [sont] réduites et la réduction consolidée $»^{35}$. En quelques années, il réussit à imposer le caractère curable de cette pathologie. 

(déformation sinueuse et permanente de la colonne vertébrale avec rotation des vertèbres). Il donne, préalablement au traitement, des soins par des bains de vapeur, une application d'émollients toniques ou fortifiants, des douches, des massages et des pressions manuelles sur les zones saillantes. Puis il utilise ses lits et fauteuils, avec une base commune sur laquelle des "modules» peuvent être ajoutés afin de les personnaliser en fonction de la morphologie de chaque patient. La reprise de la marche se fait à l'aide de béquilles spécifiques, usage qui semble être assez novateur à l'époque.

François Humbert n'est pas le seul médecin à avoir réalisé des modèles réduits de ses "machines» thérapeutiques. Le docteur Pravaz ${ }^{36}$, de Lyon, un de ses contemporains, également spécialisé dans l'orthopédie et en particulier dans la LCH, confectionne de la même manière des maquettes de ses appareils. Cinq d'entre elles sont au musée d'Histoire de la médecine de Lyon ${ }^{37}$. Dans une démarche comparable à celle de François Humbert, le docteur Charles-Gabriel Pravaz les présente avec son mémoire sur l'orthopédie ${ }^{38}$, le 26 août 1844, à la Société de chirurgie de Lyon. Son fils Jean Charles Théodore ${ }^{39}$ les expose à nouveau le 17 février 1864 à la Société de chirurgie de Paris, en même temps qu'il fait découvrir son mémoire sur la Curabilité des luxations congénitales $d u$ fémur ${ }^{40}$. On peut également citer les modèles réduits du colonel Amoros, exposés à côté de ceux de François Humbert lors de l'Exposition des produits de l'industrie française de 1834 avec une mise en scène de personnages en carton, en situation d'exercice de rééducation sur des appareils ${ }^{41}$. Plus tardivement, des maquettes de l'Institut orthopédique de New York sont exposées à Paris, à l'occasion de l'Exposition universelle de 1867 , où des structures de santé sont représentées ${ }^{42}$.

\section{Conservation et valorisation des maquettes de François Humbert}

\section{Les vicissitudes de la collection de maquettes depuis 1850}

Trente-huit à quarante maquettes ${ }^{43}$ figurent parmi la collection d'œuvres et d'objets d'art donnés à la Ville de Bar-le-Duc par la belle-fille et unique héritière de François Humbert. Fabriquées au sein de l'établissement orthopédique de Morley selon les plans et élévations géométrales du médecin orthopédiste, elles servent non pas de prototypes mais plus vraisemblablement de modèles réduits d'exposition des bâtis de lits, fauteuils et appareils auxiliaires adaptés à chaque difformité congénitale ou luxation ancienne. Ces modèles sont les seuls exemples connus de réalisations d'appareils orthopédiques à échelle 1 qui auraient disparu au cours du $\mathrm{xx}^{\mathrm{e}}$ siècle ${ }^{44}$. Maquettes à vocation utilitaire, elles sont néanmoins considérées comme objets de collection par leur inventeur luimême puisqu'un "musée orthopédique " les expose à côté des ateliers techniques de l'établissement de soins ${ }^{45}$.

À l'été 1850, après le décès de François Humbert, le maire de Bar-le-Duc, Bernard Trichon-Saint-Paul, sollicite officiellement son unique héritière afin d'enrichir les collections du musée municipal. L'établissement, créé quelques années auparavant et installé dans l'hôtel de Florainville (actuel tribunal de grande instance), est placé sous la responsabilité de l'architecte départemental Théodore Oudet, véritable et laborieux artisan de ce don particulier. En effet, il ne faut pas moins de six mois et de nombreux échanges épistolaires entre la Ville et la belle-fille du médecin pour que la donation se 
concrétise : des réticences, dont les causes restent obscures, se manifestent au niveau des plus hautes instances municipales. Pensant que la prise en charge des maquettes par la Ville de Bar-le-Duc n'aboutirait jamais, Mme Veuve Humbert les propose à des tiers, comme le Musée lorrain de Nancy. L'un des modèles orthopédiques les plus insignes, «l'hybomètre ", échoit ainsi au musée de l'École de médecine de Nancy ${ }^{46}$. Malgré tout, le reste de la collection de maquettes rejoint le patrimoine de Bar-le-Duc à la suite de nouvelles mises en garde de Théodore Oudet au conseil municipal ${ }^{47}$. Le 23 décembre 1850, convoyées par le voiturier de Morley, dix caisses de maquettes orthopédiques et vingt caisses de "pièces d'histoire naturelle " passent l'octroi en direction du musée de Bar-le-Duc ${ }^{48}$.

Le musée, émanation d'une société savante divisée en trois sections (archéologie, arts, sciences naturelles), est administré par une commission qui publie son premier catalogue en $1880^{49}$. À cette date, si d'autres dons de Mme Humbert figurent en bonne place parmi les pièces du "jardin archéologique », de la salle d'armes, ou parmi les tableaux de la "galerie des illustrations du département de la Meuse » (personnages célèbres), ni le portrait du médecin orthopédiste (fig. 18), ni ses inventions mécaniques ne sont exposées. Au nombre des « illustrations civiles » du département, seul parmi les représentations de députés ou pairs de France, magistrats, préfets ou évêques, se trouve exposé le portrait d'un mécanicien contemporain de François Humbert, Jean Werly, inventeur des corsets sans couture, par ailleurs aïeul de l'un des membres les plus influents de la commission du musée. Il semble que les quarante maquettes orthopédiques restent ainsi durant trente ans à l'abri des regards, peut-être scellées dans leurs caisses d'acheminement originelles, et sans doute conservées dans les caves ou les combles du musée. 
Figure 18

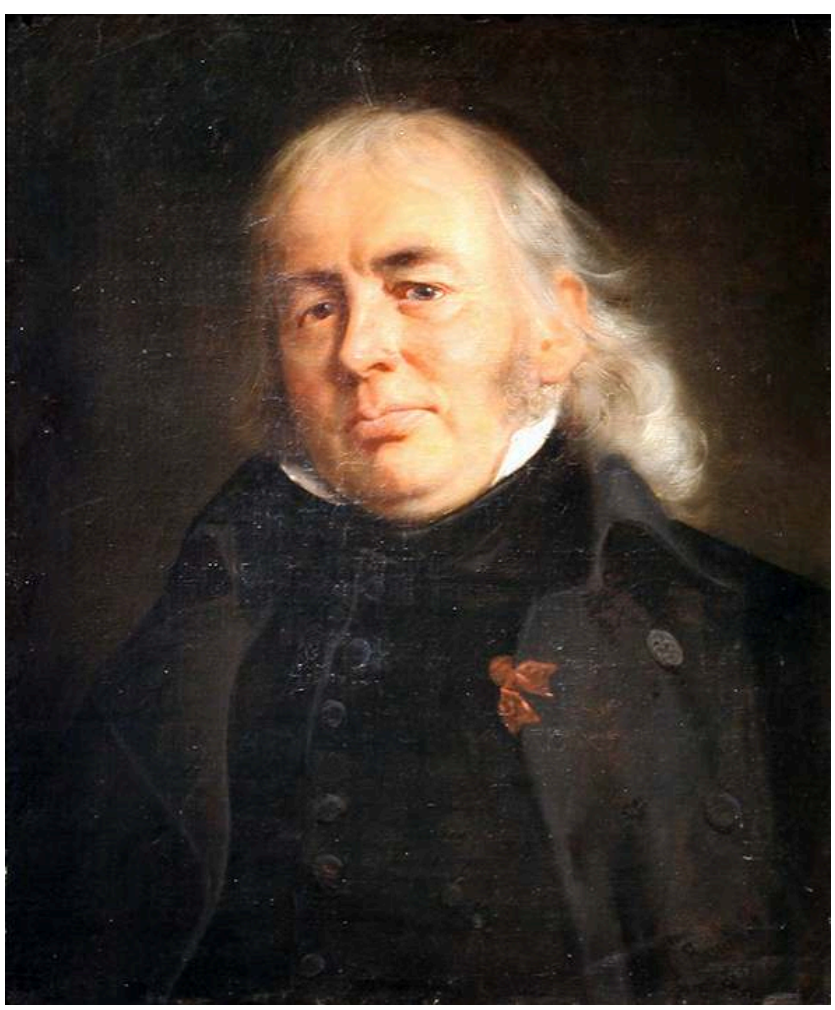

Louis BOUCHOT (1817-1860), «Portrait de François Humbert ». Huile sur toile, XIXe siècle. Collection du Musée barrois, Legs Mme Veuve Humbert, 1893. n inv. 893.35.

(c) Musée barrois, Bar-le-Duc, 2009.

En septembre 1890, les maquettes sont déposées par le musée au «Musée géographique, ethnographique, industriel et commercial " inauguré en 1885 dans les locaux de l'ancien collège Gilles-de-Trèves ${ }^{50}$ à Bar-le-Duc. Ce musée, communément appelé « musée de Géographie », est administré par la section meusienne de la Société géographique de l'Est, et entend valoriser et exposer au public le fruit de recherches, collectes et acquisitions dans des domaines aussi variés que les sciences géographiques, géologiques et statistiques, l'histoire naturelle, l'ethnographie naissante ; s'y ajoutent les produits des innovations scientifiques, artisanales, industrielles et commerciales touchant au territoire meusien. De nombreux envois des ministères de l'Intérieur, des Colonies et de la Marine, ainsi que les dépôts du musée du Trocadéro, complètent l'ensemble. On ne distingue nulle maquette de François Humbert dans les rares clichés des salles du premier musée de Géographie (fig. 19). Cependant, en 1891, l'ensemble est déménagé en Ville Haute de Bar, dans les salles d'un ancien casernement de soldats vétérans. Là, le témoignage d'un professeur du lycée de Bar-le-Duc confirme, sinon l'exposition des maquettes, du moins leur présence. Le visiteur indique que les maquettes sont «tellement délabrées, malgré un premier nettoiement fait aux frais de la Société de géographie, qu'il faudrait à la commission dudit musée des ressources qu'elle est loin de posséder pour les remettre en bon état. L'appel à la générosité des habitants de la ville de Bar, pour l'aider dans sa mission, [...] a [...] peu de succès $»^{51}$. 


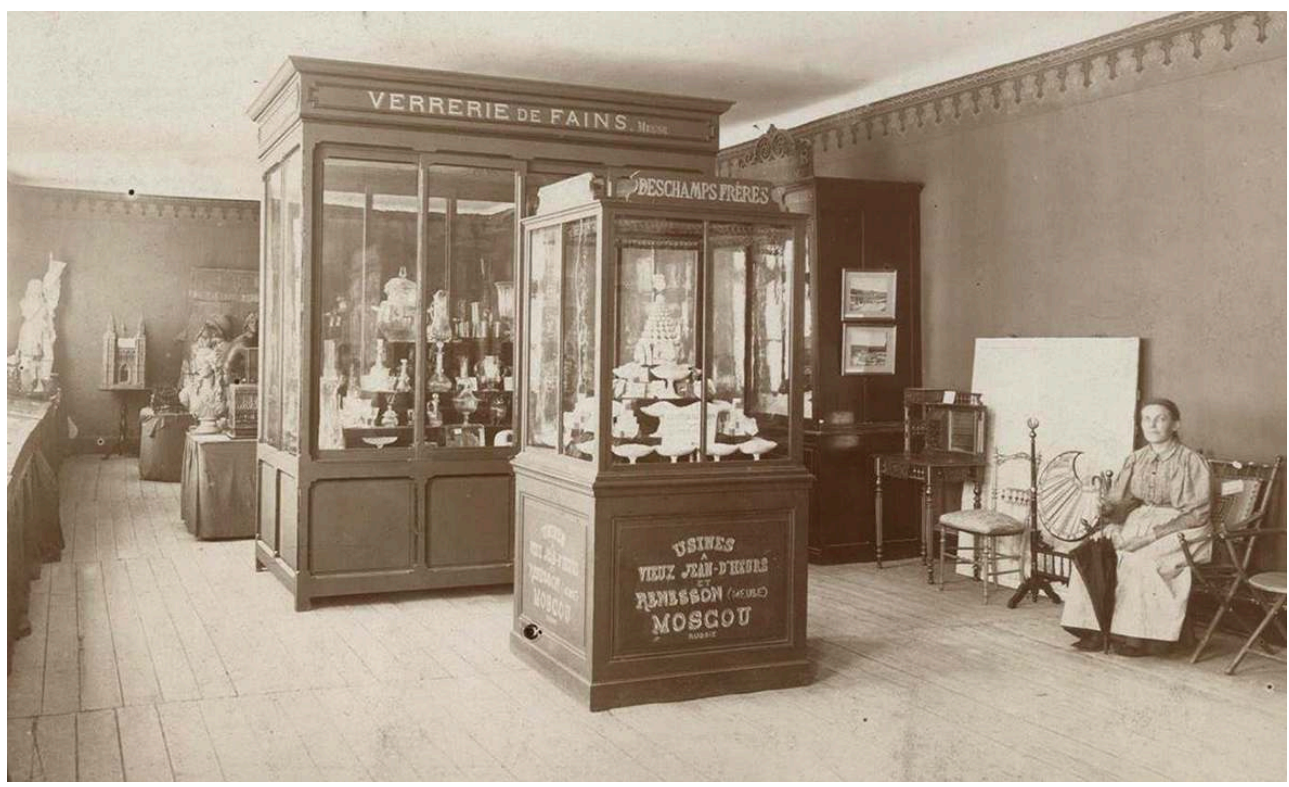

Vue d'une salle du Musée de Géographie. Tirage sur papier albuminé, entre 1885 et 1896 . Collection du Musée barrois, fonds ancien. $n^{\circ}$ inv. prov. 13.07.01.14.

(c) Musée barrois, Bar-le-Duc, 2013.

En 1903, la section meusienne se sépare de la Société de géographie de l'Est qui siège à Nancy. Les collections du musée de Géographie sont mises en caisses trois ans plus tard, à l'occasion de la vente des anciennes halles par la municipalité. Elles sont conservées jusqu'en 1908 par le propriétaire des bâtiments et président de la nouvelle Société de géographie, le docteur Voirin. Ce dernier conçoit alors une exposition renouvelée des collections, au deuxième étage de l'hôtel de ville de Bar-le-Duc, dans l'ancien hôtel particulier du maréchal Oudinot. L'inauguration, le 18 octobre 1913, donne à voir, notamment, les «véritables chefs-d'œuvre d'ébénisterie " que sont les maquettes de François Humbert ${ }^{52}$, aux côtés des produits de l'artisanat et de l'industrie locaux: verrerie, papeterie, bleuterie, ébénisterie, confiserie, corsetterie et bonneterie, lunetterie. Les maquettes sont ainsi exposées au public, le dimanche après-midi, jusqu'au début de la Deuxième Guerre mondiale: l'absorption de la Société de géographie par la Société des lettres, sciences et arts de Bar-le-Duc, en 1925, ne modifie pas la distinction administrative et physique entre les collections du musée de Géographie et celles du musée municipal.

41 Le 30 août 1939, le maire de Bar-le-Duc demande à la Société des lettres de faire évacuer les locaux de toute urgence pour les besoins de l'administration militaire. Les maquettes sont probablement mises en caisses et déplacées à cette occasion, puisqu'elles n'ont pas à souffrir de l'incendie qui touche, le 25 avril 1944, le deuxième étage de l'hôtel de ville et détruit une partie non négligeable des collections laissées sur place, ainsi que l'intégralité des vitrines historiques.

Si les collections du musée municipal de Bar-le-Duc, évacuées de l'hôtel de Florainville en 1939, sont partiellement redéployées après 1945 au premier étage de l'hôtel de ville, les collections du musée de Géographie évacuées en 1939 restent en caisses, faute de vitrines $^{53}$. De septembre 1939 à janvier 1958, leur lieu de conservation est incertain. On sait que trente-quatre maquettes sont déposées à l'hôpital-hospice de Bar-le-Duc à cette période. Conservées dans des bâtiments inaugurés en 1913 dans le quartier 
Couchot, elles sont peut-être sorties de caisse à des fins d'étude - le plan de répartition pavillonnaire de l'hôpital-hospice ne laissant apparaître aucun espace d'exposition d'objets patrimoniaux ${ }^{54}$.

En 1958, l'établissement hospitalier, engagé dans d'importants travaux de réfection, remet les maquettes au conservateur des Musées de la Meuse, François Pomarède, «à des fins de restauration et d'exposition ${ }^{55} »$. En effet, la municipalité entreprend au même moment une réflexion globale de conservation et mise en valeur des collections patrimoniales barisiennes au sein d'un musée rénové. La Ville prend alors en charge les collections du musée de Géographie que la Société des lettres n'avait plus les moyens de conserver ni de présenter. Matériellement, l'ensemble des collections de l'ancien musée de Géographie est alors déplacé dans l'une ou l'autre des réserves temporaires du musée municipal, disséminées dans les caves du château de Marbeaumont, dans celles du tribunal, dans les galeries de l'église Saint-Jean et dans une annexe proche de l'hôtel de ville, rue Werly.

Le transfert de propriété des maquettes est officialisé en 1966, sous la supervision du conservateur qui mène à terme le projet amorcé, Émile Meslé. Révélateur des intérêts scientifiques des années 1960-1970, l'intégralité des collections ethnographiques et d'histoire naturelle sont alors inscrites à l'inventaire réglementaire du musée municipal, tandis que les maquettes de François Humbert, comme d'autres "inventions", productions artisanales et industrielles de l'ancien musée de Géographie, sont enregistrées en lots non détaillés et administrativement gérées comme du matériel documentaire.

Cependant, les maquettes font partie intégrante du programme muséographique en cours de définition. Au projet d'installation du futur « Musée barrois » dans le collège Gilles-de-Trèves, abandonné au début des années 1960, succède le projet d'aménagement des vestiges de l'ancien château des ducs de Bar. La distribution des collections présentée à la commission du musée de Bar-le-Duc en 1969 prévoit, dans la salle "Arts et traditions populaires ", sept sections ${ }^{56}$ parmi lesquelles, dans la section "Les inventions", quelques maquettes, exposées aux côtés d'une maquette de la machine de Cugnot, de trois vélocipèdes Michaux et de corsets sans couture. Il semble que ce projet se soit concrétisé, comme en témoignent aujourd'hui les visiteurs du musée qui ont connu les salles inaugurées en 1974. Cependant, aucune photographie n'en garde trace, et aucun document concernant la restauration des maquettes n'a été conservé.

En effet, quelques maquettes de lits et de fauteuils comportent aujourd'hui des pièces de textile et de bois blanc qui trahissent une consolidation relativement récente. Peutêtre sont-elles ajoutées à l'occasion de l'une des expositions documentées dans les années 1980. Ainsi, le 19 juin 1982, le Musée barrois prête cinq maquettes pour une journée d'exposition consacrée au docteur Humbert au collège André Theuriet de Barle-Duc. En 1984, c'est l'ALCIDE, association de loisirs et culture cantonale, qui présente à Morley quatre maquettes dans le cadre de la Fête des moissons.

La muséographie du Musée barrois est profondément remaniée dans les années 1990, s'orientant vers la mise en valeur de l'archéologie, des beaux-arts et du patrimoine militaire, et accroissant sa surface d'exposition temporaire. Les maquettes de François Humbert sont alors remisées dans le grenier du musée, empilées dans des sacs poubelle non fermés. Ce n'est qu'en 2013 que quelques maquettes sont à nouveau exposées au public, à l'initiative du Cercle généalogique de la Meuse (Journées d'histoire régionale à 
l'abbaye de Pont-à-Mousson). L'équipe du Musée barrois, épaulée par l'Association François Humbert de Morley et par l'expertise d'un étudiant interne en médecine orthopédique, Antoine Desseaux, prend alors les mesures conservatoires qui s'imposent : la collection est entièrement déployée, pré-inventoriée, et bénéficie d'une campagne photographique. Chaque maquette ou élément désolidarisé est comparé avec les plans et élévations dressés par leur inventeur, avant un reconditionnement soigneux de la collection, en attendant une campagne de restauration globale.

\section{L'actuelle renaissance de la collection} mécanismes structurant ces objets, il s'agit plutôt de restituer leur lisibilité et de les protéger de nouvelles dégradations éventuelles. Aussi un premier travail a-t-il consisté en un pré-inventaire et un conditionnement de chacune des maquettes dans des caisses adaptées. Il a permis de réaliser un constat d'état sanitaire global. Eu égard aux conditions de stockage de ces dernières décennies, leurs structures sont dans un relatif bon état grâce à la qualité des matériaux constitutifs et de l'exécution des assemblages. Les causes des dégradations sont essentiellement dues à l'environnement humide et poussiéreux, ainsi qu'aux multiples déplacements et manipulations. Ainsi, l'ensemble des pièces présente un empoussièrement important : teintes grises des textiles, taches sombres. Des traces de moisissure apparaissent tant sur les bois exotiques (dépôts blanchâtres) que les textiles anciens ou le vernis des parties métalliques. Plusieurs zones montrent également des points d'oxydation. De plus, des déformations, des éléments décousus, des désassemblages par perte d'adhésif ou de goupille, des fentes et des pertes de bois fragilisent la structure de chaque maquette. Enfin, différentes interventions antérieures, en ajoutant des pièces en bois hétérogènes (hêtre, peuplier et contre-plaqué) ou des éléments textiles modernes, perturbent la lisibilité et la cohérence de chaque maquette. Les maquettes comprenaient à l'origine des pastilles permettant d'identifier les éléments les composant à partir des planches gravées. Beaucoup de ces pastilles ont malheureusement disparu aujourd'hui.

Aussi la restauration, la plus minimaliste possible, doit-elle améliorer les conditions de conservation de la collection, préserver les matières, rendre leur intégrité aux objets en solidarisant les éléments détachés ou cassés et permettre une meilleure lisibilité et compréhension des surfaces en présence. Les parties textiles seront nettoyées notamment par gommage. Elles seront remises en forme ou reprises par couture le cas échéant. Les ajouts textiles contemporains injustifiés du point de vue scientifique seront retirés. Les parties en bois seront également dépoussiérées et recevront un traitement antifongique. Les assemblages seront repositionnés par le biais d'adhésifs ou de chevilles métalliques. Fentes et fissures seront colmatées avec un bois de la même espèce (acajou généralement). Chaque partie métallique sera, dans la mesure du 
possible, démontée pour être nettoyée et subira un allégement de son vernis et de la cire. Cette intervention sur des matériaux multiples sera réalisée par une équipe pluridisciplinaire expérimentée dans la restauration d'objets patrimoniaux complexes. Pour chaque pièce, une fiche de traitement reprenant le constat d'état, le diagnostic, les objectifs de traitement, les interventions, sera fournie après traitement, ainsi que des photographies avant et après restauration. Les opérations de remise en fonctionnement des maquettes pour une exposition scientifique et médicale de la collection telle que la fabrication d'éléments manquants ou la réintégration des pièces en position orthopédique correcte se fera lors d'une seconde phase, selon les prescriptions d'un comité scientifique qui réunira le responsable scientifique des collections, l'équipe de restaurateurs et des médecins. Cette remise en fonctionnement ne concernera que quelques pièces emblématiques susceptibles de faciliter la compréhension de l'ensemble.

51 Pour mener à bien cette opération d'envergure, dont le montant s'élève à plus de $33000 €$, le Musée barrois et l'association François Humbert de Morley, espérant une subvention importante de l'État et de la Région Grand Est, comptent également sur la participation de partenaires privés soucieux de la préservation du patrimoine hospitalier français. Avec l'aide de la Fondation du Patrimoine, un appel au mécénat populaire sera ainsi lancé en juin 2016.

Au-delà de l'échéance de 2017 qui verra l'inauguration d'une exposition rétrospective, tant sur la personnalité et la carrière de François Humbert que sur son apport à la science et à la connaissance dans des domaines aussi variés que l'orthopédie, l'archéologie ou l'histoire naturelle, il s'agit d'inscrire cette collection exceptionnelle dans un ensemble patrimonial cohérent. Longtemps délaissée, la collection enrichirait à terme le discours muséographique du musée. Ayant vocation à valoriser toutes les formes d'art et de sciences développées dans le sud du département de la Meuse au cours de son histoire, celui-ci présente des collections variées. À l'instar des collections offertes par le propriétaire initial des maquettes, le musée, fondé en 1841 dans la veine encyclopédique chère à cette époque, conserve un grand nombre d'objets pouvant relever des beaux-arts, de l'histoire, des sciences, de l'histoire naturelle, de l'ethnologie, de l'archéologie... Cette diversité se retrouve aujourd'hui dans le parcours de visite. Il serait intéressant que ces maquettes en soient un point important, mettant ainsi l'accent sur un patrimoine technique et scientifique riche. Elles pourront être rapprochées d'autres biens appartenant à l'histoire de la médecine : le musée reçut en effet plusieurs dépôts du centre hospitalier de Bar-le-Duc ainsi qu'un legs important d'un médecin du milieu du $\mathrm{xx}^{\mathrm{e}}$ siècle $^{57}$. Terre ancienne d'innovation, le Barrois a par ailleurs vu l'invention de plusieurs procédés dans des domaines variés: vélocipèdes Michaux, moteur Dyckoff-Diesel, corsets Werly. Il parait tout à fait pertinent que les maquettes qui ont fait la gloire de François Humbert et de son village de Morley rejoignent dans le parcours de visite cet ensemble cohérent. Enfin, témoin de son époque de création, le musée conserve un grand nombre d'objets du XIX ${ }^{e}$ siècle. Dans le cadre d'un parcours chronologique, cet ensemble de maquettes doit permettre de mieux comprendre la richesse et la diversité de cette période.

Dans un même objectif pédagogique, d'autres partenariats entre le musée et l'Association François Humbert de Morley sont envisagés. À partir de la collection restaurée du musée, des films d'animation pourraient être réalisés afin d'aider le public à comprendre le fonctionnement et l'utilisation de ces «machines ». Non seulement la 
mémoire de son inventeur serait restaurée mais aussi sa volonté de diffuser auprès du plus grand nombre le fruit de ses recherches médicales dont la vocation première était de soulager et améliorer le quotidien de ses contemporains. Conformément au vœu de François Humbert, connaissance et philanthropie sont au cœur de ce projet de valorisation.

\section{Annexes}

\section{L'Association François Humbert de Morley}

Créée en 2013 par un groupe de passionnés d'histoire et de médecine, l'association souhaite contribuer à la connaissance de l'œuvre de ce personnage hors du commun que fut François Humbert.

Les buts de l'association sont de :

- promouvoir le développement des travaux de François Humbert, fondateur du premier établissement d'orthopédie français à Morley (France),

- contribuer à l'histoire de l'orthopédie et à la conservation du patrimoine s'y rapportant,

- participer à la réalisation de tous travaux de recherche, de formation et d'éducation dans le domaine de l'histoire des sciences.

\section{Association François Humbert de Morley}

5720 , rue du docteur Humbert

5855290 Morley

59 assofhumbertdemorley@gmail.com

60 Page Facebook de l'association: https://www.facebook.com/Association-F-Humbertde-Morley-591126784355248/timeline/?ref=hl

\section{Le Musée barrois de Bar-le Duc}

61 Le Musée barrois est un établissement de la communauté d'agglomération de Bar-leDuc Sud Meuse. Labellisé musée de France, il est installé au sein du quartier Renaissance de Bar-le-Duc, Ville d'Art et d'Histoire, dans le château neuf édifié à partir de 1567 par le duc Charles III. Il prend appui sur les bâtiments de l'ancienne cour des comptes et sur la salle du trésor des chartes, érigés à la fin $d u x^{e}$ siècle par René II d'Anjou.

62 Des 25000 œuvres conservées, un florilège est exposé selon de grandes sections thématiques.

63 - La section d'archéologie gallo-romaine et mérovingienne regroupe des collections provenant en grande partie de Naix-aux-Forges, Bar-le-Duc et Gondrecourt. Y figurent de remarquables pièces sculptées et damasquinées.

64 - Le parcours Beaux-Arts est chronologique, du Xve au XXe siècle. Une riche collection de sculptures lorraines du XIV ${ }^{e}$ au XVII ${ }^{e}$ siècles précède un ensemble d'art peint et sculpté de la Renaissance et des périodes maniériste et baroque. La peinture du XVIII ${ }^{\mathrm{e}}$ siècle, l'art académique et le paysage au XIX ${ }^{e}$ siècle sont également bien représentés. 
65

- Une section d'ethnographie, héritière de l'ancien «musée de Géographie », met en valeurs les arts premiers d'Afrique, d'Océanie, d'Asie et des Amériques.

- Un riche fonds d'estampes, de dessins et de photographies est régulièrement exposé.

Le Musée barrois présente deux grandes expositions temporaires par an. Il organise de nombreuses activités pédagogiques, un cycle de conférences thématiques et accueille plusieurs manifestations culturelles chaque année (concerts, colloques...).

L'actualité du Musée barrois est à découvrir sur sa page officielle (http:// www.meusegrandsud.fr/vivre/vos-loisirs/ma-culture/musee-barrois.html) et sur sa page Facebook (https://www.facebook.com/Musée-Barrois-1566502896927719/ timeline/).

Musée barrois

Esplanade du château

Rue François de Guise

55000 Bar-le-Duc

Tél. 03.29.76.14.67

musee@meusegrandsud.fr

\section{NOTES}

1. - JEANNIN, Hippolyte. «Notice Biographique. François Humbert, médecin orthopédiste, fondateur de l'établissement de Morley, département de la Meuse ». Mémoires de la Société philomatique de Verdun (Meuse), 1853, tome V, p. 257.

2. - Mémoires manuscrits de François Humbert (Archives départementales de la Meuse (AD Meuse, $8 \mathrm{~J}$ 18).

3. - Attestations (AD Meuse, $8 \mathrm{~J} 18$ ).

4. - HUMBERT, François, HUMBERT, Nicolas. De l'emploi des moyens mécaniques et gymnastiques dans le traitement des difformités du système osseux. Bar-le-Duc/Paris: F. Gigault d'olincourt/J.-B. Baillière, 1831-1835. Voir le site: http://gallica.bnf.fr/ark:/12148/bpt6k56249077 [consulté le 12/10/2016].

5. - RAPOU, Toussaint. Essai sur l'atmidiatrique, ou médecine par les vapeurs. Paris : Gabon, 1819.

6. - HUMBERT, François, HUMBERT, Nicolas. Op. cit., tome 1, p. 13.

7. - QUIN, Grégory. "Le sexe des difformités et la réponse orthopédique (1819-1862) ». Genre \& Histoire, 4, printemps 2009. Voir le site: https://genrehistoire.revues.org/523 [consulté le 12/10/2016].

8. - Le Constitutionnel, 29 juin 1822.

9. - Le Journal des débats, 4 juillet 1829.

10. - HUMBERT, François, HUMBERT, Nicolas. Op. cit., tome 1, p. 221.

11. - FODÉRÉ, François-Emmanuel. Journal complémentaire du dictionnaire des sciences médicales. Paris : Panckouke, 1824.

12. - Certificat du conseil municipal de Morley, 21 juin 1835 (AD Meuse, 8 J 20). 
13. - HUMBERT, François. Notice sur les appareils et machines exposés à l'exposition publique des produits de l'industrie française inventés par le Docteur Humbert père et employés dans l'établissement orthopédique fondé par lui à Morley dès l'année 1817. Bar-le-Duc : F. Gigault d'Olincourt, 1834, p. 7.

14. - HUMBERT, Nicolas. Dissertation sur l'érysipèle. Thèse de médecine, Faculté de Paris. Paris: Didot, 1830.

15. - « Inventaire des objets donnés au Musée de Bar-le-Duc par Mme Vve Jules Humbert [...]» (Musée barrois, documentation, dossier François Humbert).

16. - Ibid.

17. - POËY d'AVANT, Faustin. Les Monnaies féodales de France [1858-1862]. Éd. Georges Depeyrot. Paris : Maison Florange, 2007.

18. - CARLINO, Andrea, JEANNERET, Michel. Vulgariser la médecine: du style médical en France et en Italie, XVI et XVII siècles. Genève: Droz, 2009 ; voir par exemple la Gazette de santé, contenant les nouvelles découvertes sur les moyens de se bien porter et de guérir quand on est malade, périodique créé par Jacques-Joseph de Gardanne, qui parut de 1773 à 1789 puis de 1804 à 1829.

19. - HUMBERT, François, JACQUIER, Nicolas. Traité des difformités du système osseux. Paris : Baillière, 1838, t. 2, p. 52-53.

20. - Le Provincial, 27 juillet 1828.

21. - Diplômes de membre correspondant (AD Meuse, $8 \mathrm{~J} 18$ ).

22. - PRAVAZ, Charles-Gabriel. «Essai ou Observations sur la manière de réduire les luxations spontanées... ». L'Expérience, 1838 ; CHELIUS, Joseph Maximilian. A System of Surgery. Philadelphie : Lee and Blanchard, 1847, p. 245 ( $1^{\mathrm{e}}$ édition en allemand, Handbuch der Chirurgie. Stuttgart: Walters, 1830-1831, traduit en français en 1835-1836).

23. - Note à l'attention du conseil municipal de Bar-le-Duc, par Théodore Oudet, décembre 1850 (Musée barrois, documentation, dossier François Humbert).

24. - HUMBERT, François, HUMBERT, Nicolas. Op. cit., tome 1, p. 18.

25. - Ibid.

26. - La vérité sur les progrès récens de l'orthopédie... par un docteur en médecine de la Faculté de Paris. Paris: Feugueray, 1826, p. 8. Voir le site: http://gallica.bnf.fr/ark:/12148/bpt6k5742300f [consulté le 14/10/2016].

27. - Lettre de l'Institut de France, Académie royale des sciences, 25 juillet 1836, signée Flourens (AD Meuse, $8 \mathrm{~J}$ 19).

28. - HUMBERT, François, HUMBERT Nicolas. Op. cit., tome 1, p. 259.

29. - Voir dans la base Leonore : notice http://www.culture.gouv.fr/public/mistral/leonore_fr? ACTION=RETROUVER\&FIELD_1=NOM\&VALUE_1=HUMBERT\&NUMBER=54\&GRP=0\&REQ=\%28\%28HUMBERT\%29\%20\%3aNOM\%20\%29\&US [consulté le 14/10/2016].

30. - Musée d'anatomie pathologique créé en 1835 en l'honneur de Guillaume Dupuytren, anatomiste et chirurgien français. Il se situe 15 rue de l'École-de-médecine à Paris.

31. - Lettre du docteur Orfila à François Humbert, 1836 (AD Meuse, $8 \mathrm{~J}$ 18).

32. - JEANNIN, Hippolyte. Art. cit., p. 257.

33. - Ibid.

34. - HUMBERT, François, HUMBERT, Nicolas. Op. cit., tome 1, p. 259.

35. - HUMBERT, François, JACQUIER, Nicolas. Essai et observations sur la manière de réduire les luxations spontanées ou symptomatiques de l'articulation ilio-fémorale. Bar-le-Duc: F. Gigault d'Olincourt, 1835, p. V.

36. - Voir le site : http://cths.fr/an/prosopo.php?id=100448 [consulté le 14/10/2016].

37. - MOUNIER-KUHN, Alain. La Revue lyonnaise de médecine. Numéro spécial du 30 décembre 1966, t. $15, \mathrm{n}^{\circ} 20$, p. 942.

38. - PRAVAZ, Charles-Gabriel. Mémoire sur la réalité de l'art orthopédique et ses relations nécessaires avec l'organoplastie. Lyon : Société de médecine de Lyon, imp. de Marle, 1845.

39. - Voir le site : http://cths.fr/an/prosopo.php?id=111497 [consulté le 14/10/2016]. 
40. - PRAVAZ, Jean Charles Théodore. "La Curabilité des luxations congénitales du fémur». Bulletin de la Société de chirurgie de Paris, $1865,2^{\mathrm{e}}$ série, $5^{\mathrm{e}}$ tome, p. 38.

41. - Gazette médicale de Paris, 1834, p. 339-340.

42. - Exposition universelle de 1867 à Paris. Catalogue général publié par la Commission impériale. Paris/ Londres : Dentu/J. M. Johnson and sons, 1867, vol. 1, p. 17 et dans le catalogue spécial p. 56.

43. - La difficulté de reconstitution des maquettes altérées explique l'approximation du dénombrement actuel.

44. - DELATTE, Pascal. Vie et œuvre du docteur François Humbert (1776-1850). Thèse de doctorat en médecine. Nancy : université de Nancy I, 1982, p. 25.

45. - Ibid., p. 25.

46. - Ibid., p. 42.

47. - Note [...] par Th. Oudet, décembre 1850. Op.cit. (Musée barrois, documentation, dossier François Humbert).

48. - Bordereau d'expédition adressé par Mme Humbert au musée de Bar-le-Duc, 23 décembre 1850 (Musée barrois, documentation, dossier François Humbert).

49. - JACOB, Alfred. Musée de Bar-le-Duc. Catalogue sommaire ou Guide du visiteur dans les différentes salles de cet établissement et dans la galerie des illustrations de la Meuse. Bar-le-Duc: Veuve Numa Rolin, Chuquet et Cie, 1880.

50. - « Registre d'inventaire du musée de Géographie : Objets provenant du musée municipal de Bar-le-Duc et déposés le 21 septembre et 10 octobre 1890 au Musée géographique, ethnographique, industriel et commercial », p. 66 (Musée barrois, documentation, dossier musée de Géographie).

51. - DESPIQUES, Paul. Une visite aux Musées de Bar-le-Duc. S.l.n.d. (vers 1896), p. 5.

52. - SIMON, J.-M. « Jeudi 23 octobre 1913. Une inauguration », coupure de presse, s.l.n.d. (Musée barrois, documentation, dossier musée de Géographie).

53. - Lettre du président de la Société des lettres, sciences et arts de Bar-le-Duc au préfet de la Meuse, 4 décembre 1950 (Musée barrois, documentation, dossier musée de Géographie).

54. - MASSON-MERCIER, Anne. L'Hôpital Saint-Denys de Bar-le-Duc. De sa fondation à nos jours. Bar-leDuc: Imprimerie du Barrois, 1985, p. 72-82 (issu d'une thèse de doctorat en médecine, Nancy 1, 1985).

55. - Décharge adressée par François Pomarède au directeur de l'hôpital de Bar-le-Duc, 23 juillet 1958 (Musée barrois, documentation, dossier objet 13.10.05.1 à 38).

56. - Extrait du registre de la commission du musée de Bar-le-Duc, 29 janvier 1969. Les sections sont ainsi définies : agriculture ; artisanat rural ; artisanat citadin ; de l'atelier à la manufacture et à l'industrie ; les inventions ; les passe-temps d'autrefois ; le folklore religieux (Musée barrois, documentation, dossier Musée barrois).

57. - Legs de Lucien Gelly en 1971 comprenant, outre des œuvres d'art, le contenu de son cabinet médical.

\section{RÉSUMÉS}

L'orthopédie connaît son essor au début du XIX siècle. Ceux qui étaient appelés les «boîteux » et les «bossus » voient apparaître des traitements se situant entre avancées scientifiques et progrès techniques. L'essentiel de leur prise en charge se fait par des moyens mécaniques, des 
«machines » constituées de lits et de fauteuils pourvus de systèmes de traction. François Humbert (1776-1850), médecin de campagne et collectionneur, fonde en 1817, à Morley (Meuse) le premier établissement orthopédique de France, qui accueille environ trois cents patients. Dans cet établissement éloigné des centres universitaires, il fut le premier à se pencher sur le traitement des luxations congénitales de la hanche, réputées incurables. Détaillées dans un ouvrage expliquant le fonctionnement de ces inventions, une quarantaine de maquettes de belle facture sont utilisées pour diffuser ces travaux novateurs et en favoriser la reconnaissance institutionnelle ainsi que la vulgarisation auprès du public. La collection a connu une histoire mouvementée avant de rejoindre le Musée barrois. Aujourd'hui, un projet de conservation préventive et de restauration s'élabore, grâce au partenariat entre le musée et une association. Au-delà d'un projet d'exposition célébrant le bicentenaire de la création de l'établissement de Morley, en 2017, une réflexion a été engagée à propos de l'inscription de l'ensemble, très spécifique, dans la muséographie des salles d'exposition permanente, en lien avec le patrimoine technique et scientifique meusien.

The early nineteenth century can be considered as a turning point in orthopaedics. Scientific and technical progress allowed those who were called 'lame' and 'hunchbacks' to be given new treatments. These new cures were very much based on mechanical appliances, 'machines' which consisted of beds and chairs incorporating traction systems. In 1817, in Morley (Meuse department), François Humbert (1776-1850), a country doctor and collector, created the first French orthopaedic institution, which accommodated about three hundred patients. In an establishment far from academic centres, he was the first doctor to examine the possibility of treating congenital hip dislocation, deemed incurable at that time. Supported by a book which explains the operation of his inventions, about forty well-made models constitute an element of communication about this innovative work, well worth better institutional recognition and accessibility to the public. The collection had a turbulent history before arriving in the Barrois museum in the city of Bar-le-Duc. Today, a project of preventive conservation and restoration is being developed through a partnership between the museum and an association. Beyond a project of an exhibition to celebrate the bicentennial of the creation of the Morley institution in 2017, reflection has been initiated concerning the inclusion of this highly specific collection in the museum presentation of the permanent exhibition rooms, in the broader context of the technical and scientific heritage of the Meuse department.

\section{INDEX}

Keywords : Orthopaedics, models, medicine, collection, museum, restoration, preventive conservation, Meuse, Bar-le-Duc, Morley, Barrois Museum, François Humbert

Mots-clés : orthopédie, maquette, médecine, collection, musée, restauration, conservation préventive, Meuse, Bar-le-Duc, Morley, Musée barrois, François Humbert

\section{AUTEURS}

\section{ANTOINE DESSEAUX}

Chirurgien orthopédiste, service de chirurgie orthopédique et traumatologique de la Cavale blanche, CHRU Brest, France, antoine.desseaux@chu-brest.fr 


\section{ÉTIENNE GUIBERT}

Conservateur du patrimoine stagiaire, ancien responsable du Musée barrois, Bar-le-Duc, etguibert@yahoo.fr

\section{MICHEL PIONNIER}

Président de l'Association François Humbert de Morley, assofhumbertdemorley@gmail.com MARGUERITE PRÉAU SIDO

Chargée des collections du Musée barrois, Bar-le-Duc, m.preau@meusegrandsud.fr 